\title{
Investigating the production of leptoquarks by means of zeros of amplitude at photon electron collider
}

\author{
Priyotosh Bandyopadhyay ${ }^{\mathrm{a}}$, Saunak Dutta ${ }^{\mathrm{b}}$, Anirban Karan ${ }^{\mathrm{c}}$ \\ Indian Institute of Technology Hyderabad, Kandi, Sangareddy, Telengana 502285, India
}

Received: 2 April 2020 / Accepted: 24 May 2020 / Published online: 26 June 2020

(C) The Author(s) 2020

\begin{abstract}
Leptoquarks belong to the possible candidates for explaining various anomalies in flavor physics. Nonetheless, their existence is yet to be confirmed on the experimental side. In this paper we show how zeros of the single-photon tree-level amplitude can be used to extract information as regards leptoquarks in the case of $e-\gamma$ colliders. A small number of standard model backgrounds keep the signal clean in this kind of colliders. Unlike other colliders, the zeros of the single-photon amplitude here depend on $\sqrt{s}$ and on the mass of leptoquark along with its electric charge. We perform a PYTHIA based simulation for reconstructing the leptoquark from its decay products of the first generation and estimating the background with luminosity of $100 \mathrm{fb}^{-1}$. Our analysis is done for all the leptoquarks that can be seen at an $e-\gamma$ collider with three different masses $(70 \mathrm{GeV}, 650 \mathrm{GeV}$ and 1 $\mathrm{TeV}$ ) and three different center of momentum energies (200 $\mathrm{GeV}, 2 \mathrm{TeV}$ and $3 \mathrm{TeV}$ ). The effects of non-monochromatic photons on the zeros of the amplitude under laser backscattering and the equivalent photon approximation have also been addressed.
\end{abstract}

\section{Contents}

1 Introduction . . . . . . . . . . . 1

2 Theoretical formalism . . . . . . . . . . 3

2.1 Scalar Leptoquark . . . . . . . . . . . . . . . . 3

2.2 Vector Leptoquark . . . . . . . . . . . . . . 3

3 Mass and coupling .............. . . 5

4 Leptoquark models and simulation . . . . . . . 7

4.1 Simulation set up . . . . . . . . . . . . 7

4.2 Scalar leptoquarks . . . . . . . . . . . . . . . 9

4.2.1 Leptoquark $\left(S^{+1 / 3}\right)^{c} \ldots \ldots \ldots . . .99$

\footnotetext{
a e-mail: bpriyo@phy.iith.ac.in

be-mail: ph17resch11002@iith.ac.in

c e-mail: kanirban@iith.ac.in (corresponding author)
}

4.2.2 Leptoquark $\left(R_{2}^{+2 / 3}\right)^{c} \ldots \ldots \ldots 14$

4.2.3 Leptoquark $\left(R_{2}^{+5 / 3}\right)^{c} \ldots \ldots \ldots 16$

4.2.4 Leptoquarks $\left(S_{3}^{+4 / 3}\right)^{c} \ldots \ldots \ldots . . \ldots 18$

4.3 Vector leptoquarks . . . . . . . . . . . 20

4.3.1 Leptoquark $\left(U_{1 \mu}^{+2 / 3}\right)^{c} \ldots \ldots . . .20$

4.3.2 Leptoquark $\left(V_{2 \mu}^{+4 / 3}\right)^{c} \ldots \ldots . . .22$

4.3.3 Leptoquark $\left(\tilde{V}_{2 \mu}^{+1 / 3}\right)^{c} \ldots \ldots . . .24$

4.3.4 Leptoquark $\left(U_{3 \mu}^{+5 / 3}\right)^{c} \ldots \ldots . . .26$

5 Effects of non-monochromatic photons . . . . . 28

6 Conclusion . . . . . . . . . . . . . 30

References . . . . . . . . . . . . . . 31

\section{Introduction}

Leptoquarks are proposed particles that couple to quarks and leptons simultaneously, and hence carry both non-zero baryon number and lepton number. They emerge naturally in various extensions of the Standard Model (SM), such as the Pati-Salam model [1], GUT based on $S U$ (5) or $S O(10)$ [24], extended technicolor models [5,6], etc. These color-triplet electromagnetically charged bosons (spin zero or one) could be singlet, doublet or triplet under the $S U(2)_{L}$ group [7-12]. Detection of leptoquark would be a signal for the unification of matter fields. Anomalies observed in the lepton flavor universality ratios $R_{K}, R_{K *}, R_{D}, R_{D *}$ related to rare $\mathrm{B}$ decays [13-17] and the deviations in the measurements of angular observables from their theoretical estimates can be addressed using several leptoquark models. Some of these models can explain the observed discrepancy in muon $g-2[18,19]$ and also accommodate the excess of $2.4 \sigma$ in a Higgs decay branching fraction to $\mu \tau$ at $8 \mathrm{TeV}$ with $19.7 \mathrm{fb}^{-1}$ luminosity [20]. Because of their great importance in elucidating several issues of flavor physics [21-53], leptoquarks have been studied in the literature in gory details in the last few decades 
[7-12,54-69]. In parallel, numerous searches for leptoquarks have been performed in different colliders [70-83].

On the other hand, the phenomenon of RAZ (radiation amplitude zero) was first discussed for the $q_{i} \bar{q}_{j} \rightarrow W^{ \pm} \gamma$ process at a $p p$ or $p \bar{p}$ collider in order to probe the magnetic property of the $W$-boson [84]. This phenomenon has been studied extensively in the literature for various BSM models like supersymmetry, leptoquarks, other gauge theories, and the physics behind its occurrence has also been scrutinized [85-117]. In non-Abelian theories the tree-level amplitudes ${ }^{1}$ for single-photon emission processes, which is the sum generated by attaching photon to the internal and external particles in all possible ways, can be factorized into two parts: the first part contains the combination of generators of the gauge group, various kinematic invariants, charges and other internal symmetry indices, whereas the second part corresponds to the actual amplitudes of the Abelian fields containing the dependence on the spin or polarization indices $[85,86]$. The first factor goes to zero in certain kinematical zones depending on the charge and four-momenta of the external particles and forces the single-photon tree amplitudes to vanish [87]. The general criterion for tree-level single-photon amplitude to vanish is that $\left(\frac{p_{j} \cdot k}{Q_{j}}\right)$ must be the same for all the external particles (other than photon) involved in the process [87] where $p_{j}^{\mu}$ and $Q_{j}$ are the four-momentum and charge of the $j$ th external particle and $k^{\mu}$ is the four-momentum of photon. For the $2 \rightarrow 2$ scattering processes with a photon in the final state, this condition reduces to

$$
\cos \theta^{*}=\frac{Q_{f_{2}}-Q_{f_{1}}}{Q_{f_{2}}+Q_{f_{1}}}
$$

where $Q_{f_{1}}$ and $Q_{f_{1}}$ are the charges for the incoming particles $f_{1}$ and $f_{2}$ and $\theta^{*}$ is the angle between photon and $f_{1}$ in the center of momentum (CM) frame at which RAZ occurs provided that the masses of colliding particles are negligible with respect to the total energy of the system, i.e. $\sqrt{s}$.

Linear colliders in the range of a few hundred $\mathrm{GeV}$ to 1.5 $\mathrm{TeV}$ are going to be built in the near future. These colliders can provide the possibility for studying electron-photon interactions at very high energy [118-127]. Using modern laser technology, high energetic photons with large luminosity can be prepared through laser backscattering for this kind of studies. Since very few SM processes contribute to the background for these electron-photon colliders, they can reveal clean signals of leptoquarks through zeros of the treelevel single-photon amplitude [128-130]. In this paper we study this possibility in detail. The phenomenon of RAZ in various leptoquark models has already been described in the

\footnotetext{
1 The word "amplitude" in this context is synonymous to $|\mathcal{M}|^{2}$ where $\mathcal{M}$ is the matrix element for a given process.
}

literature in the context of $e-p$ colliders where the leptoquark is expected to be produced associated with a photon or where it undergoes radiative decays $[131,132]$. Though our scenario looks quite similar to it, there arises a great difference between these two colliders while considering the position of the zero amplitude in the phase space. It is evident from Eq. (1.1) that RAZ for $e-p$ colliders occurs at some particular angle between the photon and the quark which depends only on the electric charge of electron and the quark; however, we show that the same angle for the zero amplitude at $e-\gamma$ colliders depends on the mass of the leptoquark and on $\sqrt{s}$ along with the electric charge [133]. Nevertheless, the general condition for the tree-level single-photon amplitude being zero [87] still remains valid.

In this paper we analyze all kinds of leptoquarks that are going to be produced at $e-\gamma$ colliders for three different masses $(70 \mathrm{GeV}, 650 \mathrm{GeV}$ and $1 \mathrm{TeV})$ with three different center of momentum energies ( $200 \mathrm{GeV}, 2 \mathrm{TeV}$ and $3 \mathrm{TeV}$ ). Though the leptoquark with light mass seems to be ruled out, most of these analyses assume coupling of leptoquark to a single generation of quark and lepton, whereas the results from the UA2 and CDF collaborations show that there is still room for low mass leptoquark with sufficiently small couplings and appropriate branching fractions to different generations of quarks and leptons. On the other hand, the bounds on couplings and branching fractions of the higher mass leptoquarks are more relaxed. The leptoquark will eventually decay to a lepton and a quark, and hence it will produce a mono-lepton plus di-jet signal at the detector. In a PYTHIA based analysis, we reconstruct the leptoquark from the invariant mass of the lepton and one jet. Then we look for the angle between the reconstructed leptoquark and electron and construct the angular distribution which should match with the theoretical estimates. The observation of the zeros of this distribution at the theoretically predicted portion of phase space would indicate the presence of some leptoquarks. Furthermore, we study the effects of non-monochromatic photons on the zeros of angular distribution under laser backscattering and the equivalent photon approximation schemes considering the current experimental limitations of electron-photon colliders.

The paper is organized in the following way. In Sect. 2 we describe the theoretical approach to the production of scalar and vector leptoquarks at the $e-\gamma$ collider and find the conditions for the zeros of angular distribution. The experimental constraints on the mass, coupling and branching fractions of the leptoquarks are summarized in Sect. 3. In Sect. 4, we describe the simulation set up, the choice of the benchmark points and the center of momentum energies, production cross sections and branching fractions of the leptoquarks and the PYTHIA based simulation for different types of leptoquarks produced at the electron-photon collider. Section 5 deals with the effects of non-monochromatic photons on the 
Fig. 1 Feynman diagrams for $e^{-} \gamma \rightarrow q \phi^{c}$

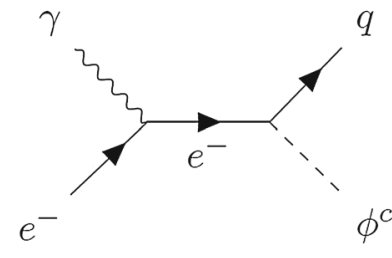

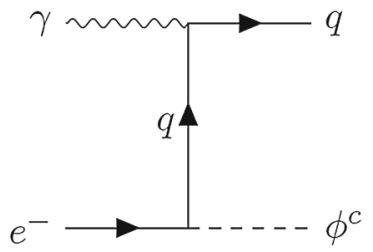

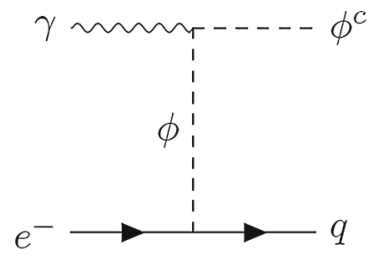

zeros of the differential distribution. Finally, we conclude in Sect. 6.

\section{Theoretical formalism}

In this section, we develop the theoretical formalism for the production of a leptoquark (more precisely, anti-leptoquark) associated with a quark or an antiquark at the electron-photon collider to get the mathematical expression for the differential distribution of this process. We consider the process $e^{-} \gamma \rightarrow$ $q \phi^{c}$ where $q$ is a quark and $\phi$ is a leptoquark (the sign ' $c$ ' indicates charge conjugate), for which there are three possible tree-level Feynman diagrams, as shown in Fig. 1.

\subsection{Scalar Leptoquark}

If the leptoquark $\phi$ is a scalar one, the matrix elements for the respective diagrams are as follows:

$$
\begin{aligned}
\mathcal{M}_{1}^{S}= & \bar{u}\left(p_{q}\right)\left(-i Y_{L}^{e q} P_{L}-i Y_{R}^{e q} P_{R}\right) \\
& \times \frac{i}{\left(\not p_{e}+\not p_{\gamma}\right)}\left(i e \gamma^{\mu}\right) u\left(p_{e}\right) \epsilon_{\mu}^{\gamma}, \\
\mathcal{M}_{2}^{S}= & \bar{u}\left(p_{q}\right)\left(-i e Q_{q} \gamma^{\mu}\right) \\
& \times \frac{i}{\left(\not p_{q}-\not p_{\gamma}\right)}\left(-i Y_{L}^{e q} P_{L}-i Y_{R}^{e q} P_{R}\right) u\left(p_{e}\right) \epsilon_{\mu}^{\gamma},
\end{aligned}
$$

$$
\begin{aligned}
\mathcal{M}_{3}^{S}= & \bar{u}\left(p_{q}\right)\left(-i Y_{L}^{e q} P_{L}-i Y_{R}^{e q} P_{R}\right) u\left(p_{e}\right) \\
& \times \frac{i}{\left(p_{q}-p_{e}\right)^{2}-M_{\phi}^{2}}\left[i e\left(1+Q_{q}\right)\left(2 p_{e}^{\mu}-2 p_{q}^{\mu}+p_{\gamma}^{\mu}\right)\right] \epsilon_{\mu}^{\gamma},
\end{aligned}
$$

where $p_{e}^{\mu}, p_{\gamma}^{\mu}$ and $p_{q}^{\mu}$ are the four-momenta of the electron, photon and the produced quark particles, respectively, $Y_{L, R}$ are $3 \times 3$ matrices describing the couplings of leptoquark with left-handed and right-handed leptons and quarks, respectively, $e$ denotes the charge of the positron, $Q_{q}$ signifies the charge of the $q$ quark in units of $e, M_{\phi}$ indicates the mass of leptoquark, $\epsilon_{\mu}^{\gamma}$ is the polarization of the photon and $P_{L, R} \equiv\left(1 \mp \gamma^{5}\right) / 2$. Here, we have deliberately neglected the masses of the electron and the quark since they would have insignificant effects on determining the zero of the amplitude involving the production of a very heavy leptoquark for all practical purposes unless the produced quark is the top quark. Therefore, after taking the spin and polarization sum of the initial and final state particles, the modulus squared matrix element for this mode becomes

$$
\begin{aligned}
& \sum_{\text {spin }}\left|\mathcal{M}^{S}\right|^{2} \\
& =\frac{e^{2}\left[\left(Y_{L}^{\mathrm{eq}}\right)^{2}+\left(Y_{R}^{\mathrm{eq}}\right)^{2}\right]\left[\left(s-M_{\phi}^{2}\right)(1-\cos \theta)+2 s Q_{q}\right]^{2}}{s\left(s-M_{\phi}^{2}\right)(1-\cos \theta)\left[s(1+\cos \theta)+M_{\phi}^{2}(1-\cos \theta)\right]^{2}} \\
& \quad \times\left[\left(s-M_{\phi}^{2}\right)^{2}(1+\cos \theta)^{2}+4 M_{\phi}^{4}\right]
\end{aligned}
$$

where $s=\left(p_{e}+p_{\gamma}\right)^{2}$ and $\theta$ is the angle between the electron and the leptoquark or equivalently the photon and the quark $q$.

\subsection{Vector Leptoquark}

Now, if the leptoquark $\phi$ is a vector particle, the matrix elements will get modified in the following way:

$$
\begin{aligned}
\mathcal{M}_{1}^{V}= & \epsilon_{v}^{\gamma} \epsilon_{\mu}^{\phi} \bar{u}\left(p_{q}\right)\left(-i Y_{L}^{\mathrm{eq}} \gamma^{\mu} P_{L}-i Y_{R}^{\mathrm{eq}} \gamma^{\mu} P_{R}\right) \\
& \times \frac{i}{\left(p_{e}+\not p_{\gamma}\right)}\left(i e \gamma^{v}\right) u\left(p_{e}\right) \\
\mathcal{M}_{2}^{V}= & \epsilon_{v}^{\gamma} \epsilon_{\mu}^{\phi} \bar{u}\left(p_{q}\right)\left(-i e Q_{q} \gamma^{v}\right) \frac{i}{\left(\not p_{q}-\not p_{\gamma}\right)} \\
& \times\left(-i Y_{L}^{\mathrm{eq}} \gamma^{\mu} P_{L}-i Y_{R}^{\mathrm{eq}} \gamma^{\mu} P_{R}\right) u\left(p_{e}\right) \\
\mathcal{M}_{3}^{V}= & \epsilon_{v}^{\gamma} \epsilon_{\mu}^{\phi} \bar{u}\left(p_{q}\right)\left(-i Y_{L}^{e q} \gamma^{\rho} P_{L}-i Y_{R}^{e q} \gamma^{\rho} P_{R}\right) u\left(p_{e}\right) \\
& \times \frac{i}{\left(p_{q}-p_{e}\right)^{2}-M_{\phi}^{2}} \\
& \times\left[i e ( 1 + Q _ { q } ) \left\{\left(2 p_{e}^{v}-2 p_{q}^{v}+p_{\gamma}^{v}\right) g_{\mu \rho}\right.\right. \\
& \left.\left.+\left(p_{q}^{\rho}-p_{e}^{\rho}-2 p_{\gamma}^{\rho}\right) g_{\mu \nu}+\left(p_{\gamma}^{\mu}-p_{e}^{\mu}+p_{q}^{\mu}\right) g_{v \rho}\right\}\right] .
\end{aligned}
$$

Here, $\epsilon_{\mu}^{\phi}$ is the polarization vector for the vector leptoquark. After taking the spin and polarization sum of the initial and 

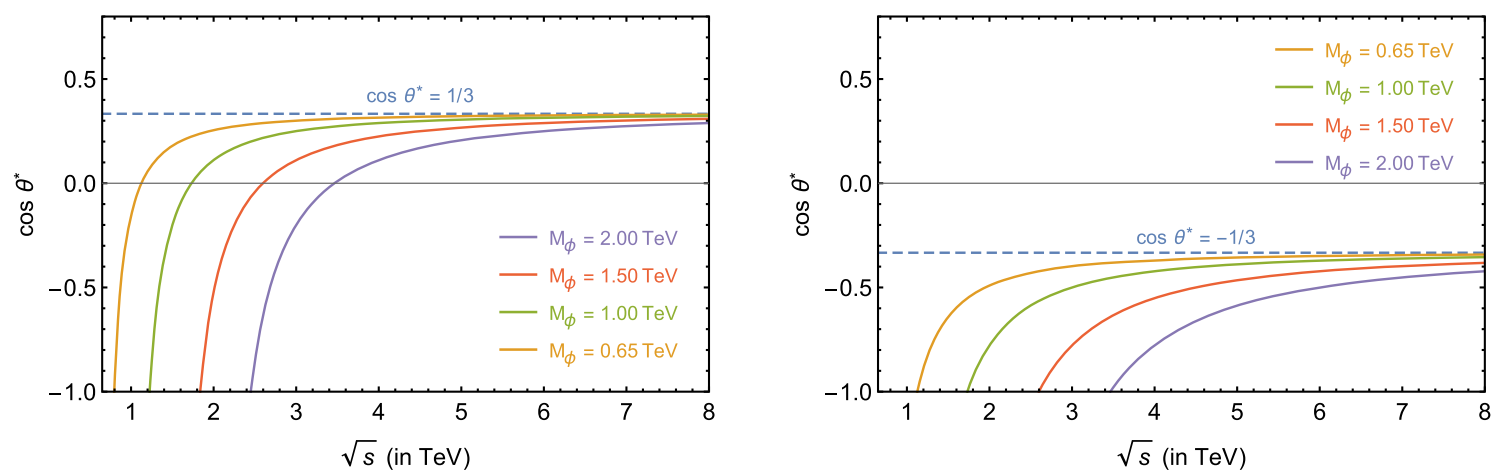

Fig. 2 Variation of $\cos \theta^{*}$ with respect to $\sqrt{s}$ for $Q_{q}=-1 / 3$ and $Q_{\bar{q}}=-2 / 3$, respectively, for different masses of leptoquark

final state particles, ${ }^{2}$ the modulus squared matrix element becomes

$$
\begin{aligned}
& \sum_{\text {spin }}\left|\mathcal{M}^{V}\right|^{2} \\
& =\frac{2 e^{2}\left[\left(Y_{L}^{\mathrm{eq}}\right)^{2}+\left(Y_{R}^{\mathrm{eq}}\right)^{2}\right]\left[\left(s-M_{\phi}^{2}\right)(1-\cos \theta)+2 s Q_{q}\right]^{2}}{s\left(s-M_{\phi}^{2}\right)(1-\cos \theta)\left[s(1+\cos \theta)+M_{\phi}^{2}(1-\cos \theta)\right]^{2}} \\
& \quad \times\left[\left\{s(1-\cos \theta)+M_{\phi}^{2}(1+\cos \theta)\right\}^{2}+4\left(s-M_{\phi}^{2}\right)^{2}\right] .
\end{aligned}
$$

The differential cross section for this process turns out to be

$$
\frac{\mathrm{d} \sigma}{\mathrm{d} \cos \theta}=\frac{s-M_{\phi}^{2}}{32 \pi s^{2}}\left(\frac{3}{4} \sum_{\text {spin }}\left|\mathcal{M}^{(S, V)}\right|^{2}\right) .
$$

Here, the one-fourth factor comes about because of the average over initial state spins and polarizations; on the other hand, the factor three indicates the number of color combinations available in the final state.

Now, it is evident from Eqs. (2.4) and (2.8) that the differential cross section vanishes iff

$$
\begin{aligned}
& \left(s-M_{\phi}^{2}\right)\left(1-\cos \theta^{*}\right)+2 s Q_{q}=0 \\
& \Longrightarrow \quad \cos \theta^{*}=1+\frac{2 Q_{q}}{\left[1-\left(M_{\phi}^{2} / s\right)\right]}=f\left(Q_{q}, M_{\phi}^{2} / s\right)
\end{aligned}
$$

since all other terms are positive quantities. This also follows from the general condition for the tree-level single-photon amplitude to vanish [87]:

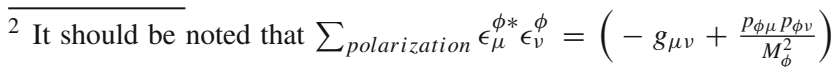
where $p_{\phi}^{\mu}$ is the four-momentum of the leptoquark.
}

$\frac{p_{e} \cdot p_{\gamma}}{-1}=\frac{p_{q} \cdot p_{\gamma}}{Q_{q}}=\frac{p_{\phi} \cdot p_{\gamma}}{Q_{\phi}}$

where $Q_{\phi}$ is the charge of leptoquark in units of $e$ and it can be expressed as $Q_{\phi}=-\left(1+Q_{q}\right)$. However, the striking difference between single-photon emission with two body final state and this process is that $\cos \theta^{*}$ in the former case does not depend on the mass of fourth particle and the center of momentum energy (as shown in Eq. (1.1)) after neglecting the masses of fermions, whereas $\cos \theta^{*}$ in the latter scenario does depend on the mass of the leptoquark and $\sqrt{s}$ (as can be seen from Eq. (2.10)). The variation of $\cos \theta^{*}$ with increasing center of momentum energy $(\sqrt{s})$ for different masses of the leptoquark is shown in Fig. 2; the left panel depicts the variation for the production of a leptoquark associated with a down-type quark, while the right panel describes the same with an up-type antiquark. It can also be observed from Eq. (2.10) that $\cos \theta^{*}$ approaches $\left(1+2 Q_{q}\right)=\left(Q_{q}-Q_{\phi}\right)$ amyptotically when $\sqrt{s}>>M_{\phi}$. For the vanishing amplitude to be inside the physical region, the condition that must be satisfied is

$Q_{q}<0 \quad$ and $\quad \frac{M_{\phi}}{\sqrt{s}} \leq \sqrt{-Q_{\phi}}$

which in turn would imply that

$-1<Q_{\phi}<0$

It should be noted that instead of quark $q$, if the leptoquark is produced with an antiquark $\bar{q}$, all the expressions for that process can be achieved by replacing $\bar{u}\left(p_{q}\right)$ with $\bar{v}\left(p_{\bar{q}}\right)$ and $Q_{q}$ with $Q_{\bar{q}}$ in the equations from Eqs. (2.1) to (2.12) where $Q_{\bar{q}}$ is the charge of $\bar{q}$ in units of $e$.

All of the leptoquarks [7-12], that can be produced at an $e-\gamma$ collider are listed in Table 1 . Here, $\Psi_{q}, \Psi_{l}$ are quark and lepton doublets whereas $q_{u}, q_{d}$ and $l_{e}$ are fields for the $u$-quark, $d$-quark and electron, respectively. The transpose $T$ acts on the $S U$ (2) indices only. $S_{3}^{a d}$ and $U_{3}^{a d}$ denote scalar and vector triplet, respectively, in the adjoint representation of SU(2); they are defined by 
Table 1 The values of $\cos \theta^{*}$ for production of different leptoquarks at $e^{-} \gamma$ collider

\begin{tabular}{|c|c|c|c|c|c|}
\hline LQ & $\mathrm{Y}$ & $Q_{e m}$ & Interaction & Process & $\cos \theta^{*}$ \\
\hline \multicolumn{6}{|c|}{ Scalar leptoquarks } \\
\hline$S_{1}$ & $2 / 3$ & $1 / 3$ & $\begin{array}{l}\bar{\Psi}_{q}^{c} P_{L} i \sigma_{2} \Psi_{l} S_{1} \\
\bar{q}_{u}^{c} P_{R} l_{e} S_{1}\end{array}$ & $\bar{u}\left(S_{1}^{+1 / 3}\right)^{c}$ & $f\left(-2 / 3, M_{\phi}^{2} / s\right)$ \\
\hline$\widetilde{S}_{1}$ & $8 / 3$ & $4 / 3$ & $\bar{q}_{d}^{c} P_{R} l_{e} \widetilde{S}_{1}$ & $\bar{d}\left(\tilde{S}_{1}^{+4 / 3}\right)^{c}$ & - \\
\hline \multirow[t]{3}{*}{$\mathbf{S}_{3}$} & $2 / 3$ & $4 / 3$ & $\bar{\Psi}_{q}^{c} P_{L}\left(i \sigma_{2} S_{3}^{a d}\right) \Psi_{l}$ & $\bar{d}\left(S_{3}^{+4 / 3}\right)^{c}$ & - \\
\hline & & $1 / 3$ & & $\bar{u}\left(S_{3}^{+1 / 3}\right)^{c}$ & $f\left(-2 / 3, M_{\phi}^{2} / s\right)$ \\
\hline & & $-2 / 3$ & & - & - \\
\hline \multirow[t]{2}{*}{$R_{2}$} & $7 / 3$ & $5 / 3$ & $\bar{\Psi}_{q} P_{R} R_{2} l_{e}$ & $u\left(R_{2}^{+5 / 3}\right)^{c}$ & - \\
\hline & & $2 / 3$ & $\bar{q}_{u} P_{L}\left(R_{2}^{T} i \sigma_{2}\right) \Psi_{l}$ & $d\left(R_{2}^{+2 / 3}\right)^{c}$ & $f\left(-1 / 3, M_{\phi}^{2} / s\right)$ \\
\hline \multirow[t]{2}{*}{$\widetilde{R}_{2}$} & $1 / 3$ & $2 / 3$ & $\bar{q}_{d} P_{L}\left(\widetilde{R}_{2}^{T} i \sigma_{2}\right) \Psi_{l}$ & $d\left(\widetilde{R}_{2}^{+2 / 3}\right)^{c}$ & $f\left(-1 / 3, M_{\phi}^{2} / s\right)$ \\
\hline & & $-1 / 3$ & & - & - \\
\hline \multicolumn{6}{|c|}{ Vector leptoquarks } \\
\hline \multirow[t]{2}{*}{$V_{2 \mu}$} & $5 / 3$ & $4 / 3$ & $\bar{\Psi}_{q}^{c} \gamma^{\mu} P_{R}\left(i \sigma_{2} V_{2 \mu}\right) l_{e}$ & $\bar{d}\left(V_{2 \mu}^{+4 / 3}\right)^{c}$ & - \\
\hline & & $1 / 3$ & $\bar{q}_{d}^{c} \gamma^{\mu} P_{L}\left(V_{2 \mu}^{T} i \sigma_{2}\right) \Psi_{l}$ & $\bar{u}\left(V_{2 \mu}^{+1 / 3}\right)^{c}$ & $f\left(-2 / 3, M_{\phi}^{2} / s\right)$ \\
\hline \multirow[t]{2}{*}{$\widetilde{V}_{2 \mu}$} & $-1 / 3$ & $1 / 3$ & $\bar{q}_{u}^{c} \gamma^{\mu} P_{L}\left(\widetilde{V}_{2 \mu}^{T} i \sigma_{2}\right) \Psi_{l}$ & $\bar{u}\left(\widetilde{V}_{2 \mu}^{+1 / 3}\right)^{c}$ & $f\left(-2 / 3, M_{\phi}^{2} / s\right)$ \\
\hline & & $-2 / 3$ & & - & - \\
\hline \multirow[t]{2}{*}{$U_{1 \mu}$} & $4 / 3$ & $2 / 3$ & $\bar{\Psi}_{q} \gamma^{\mu} P_{L} \Psi_{l} U_{1 \mu}$ & $d\left(U_{1 \mu}^{+2 / 3}\right)^{c}$ & $f\left(-1 / 3, M_{\phi}^{2} / s\right)$ \\
\hline & & & $\bar{q}_{d} \gamma^{\mu} P_{R} l_{e} U_{1 \mu}$ & & \\
\hline$\widetilde{U}_{1 \mu}$ & $10 / 3$ & $5 / 3$ & $\bar{q}_{u} \gamma^{\mu} P_{R} l_{e} \widetilde{U}_{1 \mu}$ & $u\left(\widetilde{U}_{1 \mu}^{+5 / 3}\right)^{c}$ & - \\
\hline \multirow[t]{3}{*}{$\mathbf{U}_{3 \mu}$} & $4 / 3$ & $5 / 3$ & $\bar{\Psi}_{q} \gamma^{\mu} P_{L} U_{3 \mu}^{a d} \Psi_{l}$ & $u\left(U_{3 \mu}^{+5 / 3}\right)^{c}$ & - \\
\hline & & $2 / 3$ & & $d\left(U_{3 \mu}^{+2 / 3}\right)^{c}$ & $f\left(-1 / 3, M_{\phi}^{2} / s\right)$ \\
\hline & & $-1 / 3$ & & - & - \\
\hline
\end{tabular}

$S_{3}^{a d}=\left(\begin{array}{cc}\frac{S_{3}^{+1 / 3}}{\sqrt{2}} & S_{3}^{+4 / 3} \\ S_{3}^{-2 / 3} & -\frac{S_{3}^{+1 / 3}}{\sqrt{2}}\end{array}\right) \quad$ and

$U_{3}^{a d}=\left(\begin{array}{cc}\frac{U_{3}^{+2 / 3}}{\sqrt{2}} & U_{3}^{+5 / 3} \\ U_{3}^{-1 / 3} & -\frac{U_{3}^{+2 / 3}}{\sqrt{2}}\end{array}\right)$.

\section{Mass and coupling}

The measurement of $R$-ratio from PEP and PETRA constrains the scalar leptoquarks to have $M_{\phi} \gtrsim 15-20 \mathrm{GeV}$ [67] in a model-independent way depending on the charges of them only where they are assumed to be pair-produced in the decay of a virtual photon. Measurement from AMY [70] provides $M_{\phi} \geq 22.6 \mathrm{GeV}$ for scalar leptoquarks and similar bound for vector ones too. The LEP constrains $M_{\phi} \geq 44 \mathrm{GeV}$ [71,72] with the coupling to $Z^{0}$ to be $1 / 3 \sin ^{2} \theta_{w}$ assum- ing the pair-production of leptoquarks from $Z^{0}$ and further decay of them into jets and two leptons. For decay into first two generations of quarks and leptons, this lower bound is almost independent of branching fraction; however, for the third generation there is a slight dependence. UA2 provides the relation between lowest allowed mass and the branching ratio of the leptoquark [73]. Assuming $50 \%$ branching to first generation, di-electron+ di-jet channel gives $M_{\phi} \geq 58 \mathrm{GeV}$, electron $+\not p_{T}+$ di-jet channel shows $M_{\phi} \geq 60 \mathrm{GeV}$ and combination of them provides $M_{\phi} \geq 67 \mathrm{GeV}$. However, $100 \%$ branching to first generation will exclude the mass lower than $74 \mathrm{GeV}$. DELPHI concludes $M_{\phi} \geq 77 \mathrm{GeV}$ [74], but their analysis assumes large coupling for leptoquarklepton-quark $(\lambda \geq e)$. CDF and D0 suggest the mass of leptoquarks to be greater than $113 \mathrm{GeV}$ and $126 \mathrm{GeV}$ [75], respectively, on first and second generation of leptoquarks. Several bounds from meson decays, meson-antimeson mixing, lepton flavor violating decays, lepton-quark universality, 

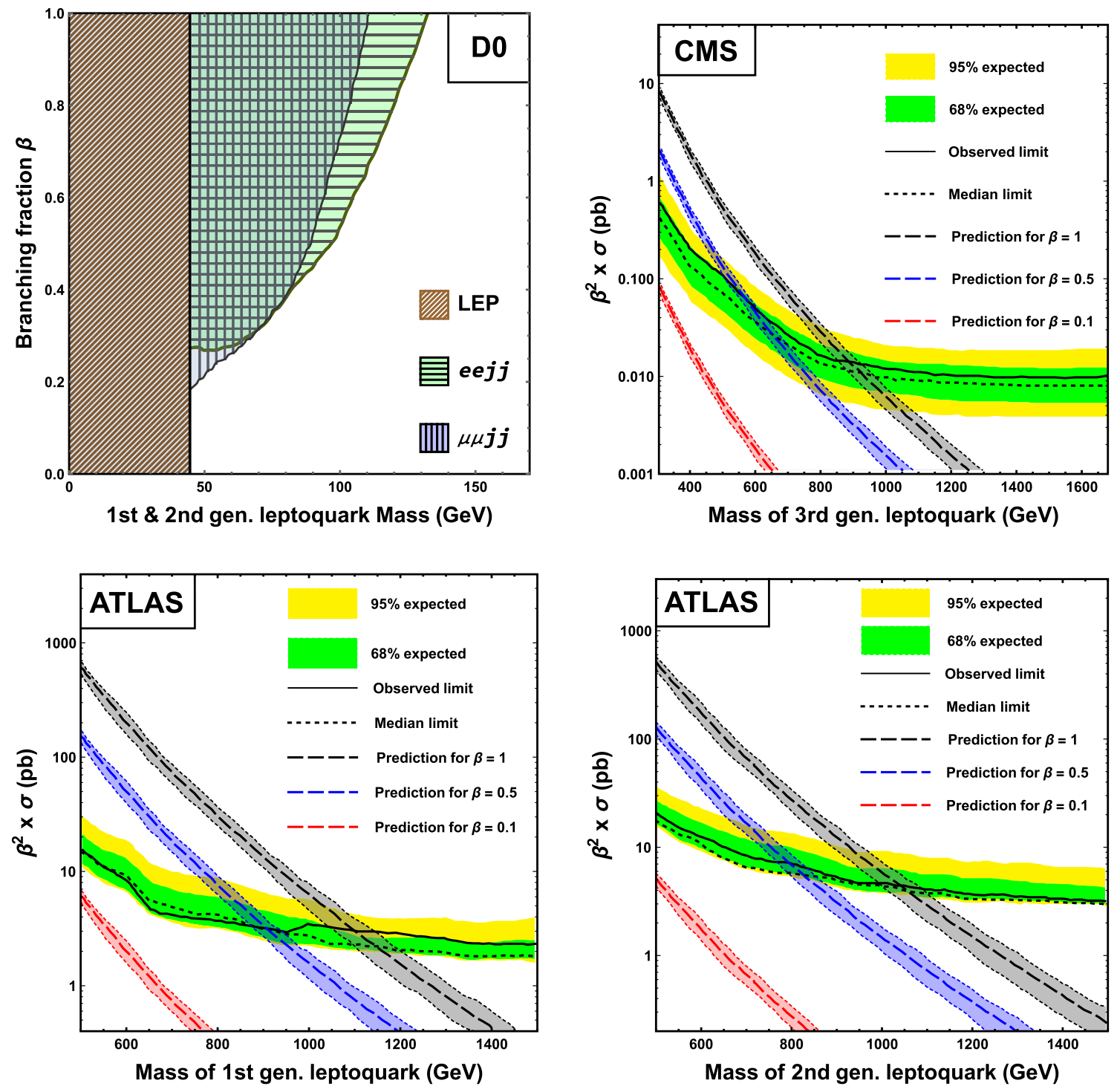

Fig. 3 Data from D0, CMS and ATLAS for the branching fraction against the allowed mass range for different generations of leptoquarks

$g-2$ of muon and electron, neutrino oscillation and other rare processes have been presented in Refs. [12,36,134-136]. If the leptoquark couples to left-handed quarks and leptons of the first generation only, then according to the pdg [136] $\lambda^{2} \leq 0.07 \times \widetilde{M}_{\phi}^{2}$ for the scalar leptoquark and $\lambda^{2} \leq 0.4 \times \widetilde{M}_{\phi}^{2}$ for the vector one where $\tilde{M}_{\phi} \equiv\left(\frac{M_{\phi}}{1 T e V}\right)$; however, the constraints change for the second generation by $\lambda^{2} \leq 0.7 \times \widetilde{M}_{\phi}^{2}$ (scalar) and $\lambda^{2} \leq 0.5 \times \tilde{M}_{\phi}^{2}$ (vector). This analysis is done for a leptoquark induced four-fermion interaction. Results from ATLAS and CMS [76-78] rule out leptoquarks with mass up to $1500 \mathrm{GeV}$ for the first and second generation leptoquarks with $100 \%$ branching and $1280 \mathrm{GeV}$ for $50 \%$ branching.

In Fig. 3, we show the plots for the branching fraction against the mass of leptoquark from Tevatron and LHC. In the top left panel, data from D0 has been presented, where the brown (obliquely meshed) region represents the disal- lowed mass range for leptoquark from LEP experiment and the greenish (horizontally meshed) and bluish (vertically meshed) areas indicate the excluded portions for the mass of the first and second generation leptoquarks from two-electron plus two-jet and two-muon plus two-jet channels at D0. The remaining three plots are from LHC for three generations of leptoquarks. The continuous black line signifies the observed limit, whereas the green and yellow areas indicate the $1 \sigma$ and $2 \sigma$ regions. The black, blue and red portions with dashed line inside show theoretical predictions with branching $(\beta)$ to be $100 \%, 50 \%$ and $10 \%$, respectively. Nevertheless, all these analyses have been done assuming that one leptoquark couples to quark and lepton from one generation only. The scenario changes drastically if branching for a leptoquark to quarks and leptons of all the generations are kept open. 
Table 2 Benchmark points for different leptoquark scenarios

\begin{tabular}{|c|c|c|c|c|c|c|c|c|}
\hline Leptoquarks & Bench-mark points & $M_{\phi}$ in $\mathrm{GeV}$ & $Y_{L}^{11}$ & $Y_{L}^{22}$ & $Y_{L}^{33}$ & $Y_{R}^{11}$ & $Y_{R}^{22}$ & $Y_{R}^{33}$ \\
\hline$\left(S_{1}^{+1 / 3}\right)^{c}$ & BP1 & 70 & 0.035 & 0.04 & 0.035 & 0.03 & 0.03 & 0.03 \\
\hline$\left(R_{2}^{+5 / 3}\right)^{c}$ & $\mathrm{BP} 2$ & 650 & 0.1 & 0.1 & 0.1 & 0.1 & 0.1 & 0.1 \\
\hline$\left(U_{1 \mu}^{+2 / 3}\right)^{c}$ & $\mathrm{BP} 3$ & 1500 & 0.1 & 0.1 & 0.1 & 0.1 & 0.1 & 0.1 \\
\hline$\left(\widetilde{R}_{2}^{+2 / 3}\right)^{c}$ & BP1 & 70 & 0.07 & 0.07 & 0.1 & - & - & - \\
\hline$\left(S_{3}^{+4 / 3}\right)^{c}$ & $\mathrm{BP} 2$ & 650 & 0.07 & 0.07 & 0.1 & - & - & - \\
\hline$\left(\widetilde{V}_{2 \mu}^{+1 / 3}\right)^{c},\left(U_{3 \mu}^{+5 / 3}\right)^{c}$ & $\mathrm{BP} 3$ & 1500 & 0.07 & 0.07 & 0.1 & - & - & - \\
\hline \multirow[t]{3}{*}{$\left(V_{2 \mu}^{+4 / 3}\right)^{c}$} & BP1 & 70 & 0.05 & 0.05 & 0.1 & 0.1 & 0.1 & 0.1 \\
\hline & BP2 & 650 & 0.05 & 0.05 & 0.1 & 0.1 & 0.1 & 0.1 \\
\hline & BP3 & 1500 & 0.05 & 0.05 & 0.1 & 0.1 & 0.1 & 0.1 \\
\hline
\end{tabular}

\section{Leptoquark models and simulation}

For our purpose, we choose four leptoquarks of different charges from the scalar sector and the same from the vector sector separately. For every leptoquark scenario, we have studied three different benchmark points (with mass $70 \mathrm{GeV}$, $2 \mathrm{TeV}$ and $3 \mathrm{TeV}$, respectively, and different couplings), each of which has been scrutinized at three distinct energy scale ( $200 \mathrm{GeV}, 2 \mathrm{TeV}, 3 \mathrm{TeV}$ ). The couplings have been picked out in such a way that they lie inside the allowed region, as shown in Fig. 3. For low mass leptoquark we use the data from D0, which allows around $25 \%$ branching to first and second generations of quarks and leptons at $M_{\phi}=70 \mathrm{GeV}$. For the heavy leptoquark scenarios, one should look at the graphs from ATLAS and CMS. There is no data for ATLAS beyond the mass range $500 \mathrm{GeV}>M_{\phi}>1.5 \mathrm{TeV}$; similarly CMS probes the mass range for the leptoquark to be $300 \mathrm{GeV}>$ $M_{\phi}>1.7 \mathrm{TeV}$.

The benchmark points used in our analysis for different leptoquarks are described in Table 2. It should be kept in mind that $\widetilde{R}_{2}, \mathbf{S}_{3}, \widetilde{V}_{2 \mu}$ and $U_{1 \mu}$ do not have any coupling to right-handed leptons. The production cross sections and branching fractions for all the leptoquarks under consideration have been put together in Tables 3 and 4, respectively. The tree-level cross sections and branching fractions have been calculated using CalcHEP 3.7.5 [137]. It should be noticed that, the mass of the leptoquark being higher than the center of momentum energy, the scenarios BP2 and BP3 cannot be explored at $\sqrt{s}=200 \mathrm{GeV}$. On the other hand, the top quark being heavier than the leptoquarks of the BP1 case, it will not get produced by decay of the latter ones. The production cross sections for the vector modes are in general higher than that of the scalar modes, which happens mainly because of two reasons. Firstly, vector leptoquarks couple to the vector currents giving rise to a very different distribution from the scalar case. Secondly, any vector leptoquark has three states of polarizations, which enhances the production cross section.

The zeros of the amplitude are shown for the leptoquarks having charges $-1 / 3$ and $-2 / 3$ only since the other ones fail to satisfy Eq. (2.13). The zeros for all these scenarios have been merged in Table 5. It should be noted that unlike BP2 and BP3 at $\sqrt{s}=200 \mathrm{GeV}$, a leptoquark of $1.5 \mathrm{TeV}$ mass (BP3) and charge $-1 / 3$ gets produced at $\sqrt{s}=2 \mathrm{TeV}$; but it does not show a zero in the distribution, since the ratio of its mass squared to $s$ is larger than its charge violating the condition in Eq. (2.12). It should also be noticed that due to the low mass of the leptoquark in BP1, $\cos \theta^{*}$ reaches the asymptotic value of $\pm 1 / 3$ at $\sqrt{s}=2 \mathrm{TeV}$ and $3 \mathrm{TeV}$ in the cases of both $Q_{\phi}$ being $-1 / 3$ and $-2 / 3$. In the next few sections, we discuss the kinematical distributions leading to appropriate cuts and final states. Subsequently, we present the signal and background number for those final states for different center of momentum energies at the integrated luminosity of $100 \mathrm{fb}^{-1}$.

\subsection{Simulation set up}

For the simulation in an electron-photon collider we implement the scenarios in SARAH 4.13 .0 [138]. Later model files are generated for CalcheP 3.7.5, which is used for signal and background event generation. The generated events have then been simulated with PYTHIA 6.4 [139]. The simulation at hadronic level has been performed using Fastjet-3.2.3 [140] with the CAMBRIDGE AACHEN algorithm. For this purpose, the jet size has been selected to be $R=0.5$, with the following criteria:

- Calorimeter coverage: $|\eta|<4.5$.

- Minimum transeverse momentum of each jet: $p_{T, \min }^{\mathrm{jet}}=$ $20.0 \mathrm{GeV}$; jets are ordered in $p_{T}$.

- Leptons $(\ell=e, \mu)$ are selected with $p_{T} \geq 10 \mathrm{GeV}$ and $\mid \eta \leq 2.5$. 
Table 3 Production cross sections for the chosen leptoquarks at $e-\gamma$ collider for the benchmark points listed in Table 2 at center of momentum energies of $200 \mathrm{GeV}, 2 \mathrm{TeV}$ and $3 \mathrm{TeV}$

\begin{tabular}{|c|c|c|c|c|c|c|c|}
\hline \multirow[t]{2}{*}{$\sqrt{s}$ in $\mathrm{TeV}$} & \multicolumn{3}{|c|}{ Cross section in $\mathrm{fb}$} & \multirow[t]{2}{*}{$\sqrt{s}$ in $\mathrm{TeV}$} & \multicolumn{3}{|c|}{ Cross section in $\mathrm{fb}$} \\
\hline & BP1 & BP2 & BP3 & & BP1 & BP2 & BP3 \\
\hline \multicolumn{4}{|c|}{ Leptoquark $\left(S^{+1 / 3}\right)^{c}$} & \multicolumn{4}{|c|}{ Leptoquark $\left(U_{1 \mu}^{+2 / 3}\right)^{c}$} \\
\hline 0.2 & 430.24 & - & - & 0.2 & 482.41 & - & - \\
\hline 2.0 & 6.61 & 50.65 & 31.95 & 2.0 & 803.82 & 58.95 & 14.84 \\
\hline 3.0 & 3.30 & 26.03 & 17.98 & 3.0 & 812.59 & 68.04 & 10.55 \\
\hline \multicolumn{4}{|c|}{ Leptoquark $\left(R_{2}^{+5 / 3}\right)^{c}$} & \multicolumn{4}{|c|}{ Leptoquark $\left(V_{2 \mu}^{+4 / 3}\right)^{c}$} \\
\hline 0.2 & 517.5 & - & - & 0.2 & 12343.51 & - & - \\
\hline 2.0 & 8.10 & 59.30 & 35.96 & 2.0 & 19110.75 & 152.70 & 15.38 \\
\hline 3.0 & 3.70 & 30.79 & 20.70 & 3.0 & 19214.64 & 181.61 & 21.40 \\
\hline \multicolumn{4}{|c|}{ Leptoquark $\left(\widetilde{R}_{2}^{+2 / 3}\right)^{c}$} & \multicolumn{4}{|c|}{ Leptoquark $\left(\tilde{V}_{2 \mu}^{+1 / 3}\right)^{c}$} \\
\hline 0.2 & 226.83 & - & - & 0.2 & 2127.02 & - & - \\
\hline 2.0 & 3.61 & 2.89 & 1.78 & 2.0 & 485.34 & 26.58 & 16.38 \\
\hline 3.0 & 1.66 & 1.49 & 1.02 & 3.0 & 477.98 & 15.46 & 9.18 \\
\hline \multicolumn{4}{|c|}{ Leptoquark $\left(S_{3}^{+4 / 3}\right)^{c}$} & \multicolumn{4}{|c|}{ Leptoquark $\left(U_{3 \mu}^{+5 / 3}\right)^{c}$} \\
\hline 0.2 & 327.44 & - & - & 0.2 & 9579.55 & - & - \\
\hline 2.0 & 5.33 & 3.95 & 2.27 & 2.0 & 11769.27 & 117.41 & 21.17 \\
\hline 3.0 & 2.43 & 2.08 & 1.36 & 3.0 & 11783.95 & 124.50 & 20.50 \\
\hline
\end{tabular}

Table 4 Branching fractions of the leptoquarks for the given benchmark points

\begin{tabular}{|c|c|c|c|c|c|c|c|}
\hline \multirow[t]{2}{*}{ Modes } & \multicolumn{3}{|c|}{ Branching fraction } & \multirow[t]{2}{*}{ Modes } & \multicolumn{3}{|c|}{ Branching fraction } \\
\hline & BP1 & BP2 & BP3 & & BP1 & BP2 & BP3 \\
\hline \multicolumn{4}{|c|}{ Leptoquark $\left(S^{+1 / 3}\right)^{c}$} & \multicolumn{4}{|c|}{ Leptoquark $\left(U_{1 \mu}^{+2 / 3}\right)^{c}$} \\
\hline ue & 0.245 & 0.229 & 0.223 & $\bar{d} e$ & 0.222 & 0.225 & 0.223 \\
\hline$c \mu$ & 0.288 & 0.229 & 0.223 & $\bar{s} \mu$ & 0.261 & 0.225 & 0.223 \\
\hline$t \tau$ & - & 0.199 & 0.218 & $\bar{b} \tau$ & 0.222 & 0.225 & 0.223 \\
\hline$d v_{e}$ & 0.141 & 0.114 & 0.112 & $\bar{u} v_{e}$ & 0.128 & 0.112 & 0.111 \\
\hline$s v_{\mu}$ & 0.185 & 0.114 & 0.112 & $\bar{c} v_{\mu}$ & 0.167 & 0.112 & 0.111 \\
\hline$b v_{\tau}$ & 0.140 & 0.114 & 0.112 & $\bar{t} v_{\tau}$ & - & 0.101 & 0.109 \\
\hline \multicolumn{4}{|c|}{ Leptoquark $\left(R_{2}^{+5 / 3}\right)^{c}$} & \multicolumn{4}{|c|}{ Leptoquark $\left(V_{2 \mu}^{+4 / 3}\right)^{c}$} \\
\hline $\bar{u} e$ & 0.458 & 0.349 & 0.336 & de & 0.278 & 0.278 & 0.278 \\
\hline $\bar{c} \mu$ & 0.542 & 0.349 & 0.336 & $s \mu$ & 0.278 & 0.278 & 0.278 \\
\hline $\bar{t} \tau$ & - & 0.302 & 0.327 & $b \tau$ & 0.444 & 0.444 & 0.444 \\
\hline \multicolumn{4}{|c|}{ Leptoquark $\left(\widetilde{R}_{2}^{+2 / 3}\right)^{c}$} & \multicolumn{4}{|c|}{ Leptoquark $\left(\widetilde{V}_{2 \mu}^{+1 / 3}\right)^{c}$} \\
\hline $\bar{d} e$ & 0.248 & 0.247 & 0.247 & ue & 0.500 & 0.261 & 0.250 \\
\hline $\bar{s} \mu$ & 0.248 & 0.247 & 0.247 & $c \mu$ & 0.500 & 0.261 & 0.250 \\
\hline $\bar{b} \tau$ & 0.503 & 0.505 & 0.505 & $t \tau$ & - & 0.478 & 0.500 \\
\hline \multicolumn{4}{|c|}{ Leptoquark $\left(S_{3}^{+4 / 3}\right)^{c}$} & \multicolumn{4}{|c|}{ Leptoquark $\left(U_{3 \mu}^{+5 / 3}\right)^{c}$} \\
\hline $\bar{d} e^{+}$ & 0.248 & 0.247 & 0.247 & $u e^{+}$ & 0.5 & 0.261 & 0.25 \\
\hline $\bar{s} \mu^{+}$ & 0.248 & 0.247 & 0.247 & $c \mu^{+}$ & 0.5 & 0.261 & 0.25 \\
\hline $\bar{b} \tau^{+}$ & 0.503 & 0.505 & 0.505 & $\bar{b} \tau^{+}$ & 0.503 & 0.505 & 0.505 \\
\hline
\end{tabular}


Table 5 Values of $\cos \theta^{*}$ corresponding to zeros of differential cross section for production of leptoquark at different center of momentum energies for various benchmark points

\begin{tabular}{|c|c|c|c|c|c|c|}
\hline \multirow[t]{3}{*}{ Benchmark points } & \multicolumn{6}{|c|}{ Values of $\cos \theta^{*}$ for zeros of $(d \sigma / d \cos \theta)$ at different $\sqrt{s}$} \\
\hline & \multicolumn{3}{|c|}{ For $Q_{\bar{q}}=-2 / 3$ or $Q_{\phi}=-1 / 3$} & \multicolumn{3}{|c|}{ For $Q_{q}=-1 / 3$ or $Q_{\phi}=-2 / 3$} \\
\hline & $0.2 \mathrm{TeV}$ & $2 \mathrm{TeV}$ & $3 \mathrm{TeV}$ & $0.2 \mathrm{TeV}$ & $2 \mathrm{TeV}$ & $3 \mathrm{TeV}$ \\
\hline BP1 & -0.52 & -0.33 & -0.33 & 0.24 & 0.33 & 0.33 \\
\hline BP2 & - & -0.49 & -0.40 & - & 0.25 & 0.30 \\
\hline BP3 & - & - & -0.78 & - & -0.52 & 0.11 \\
\hline
\end{tabular}
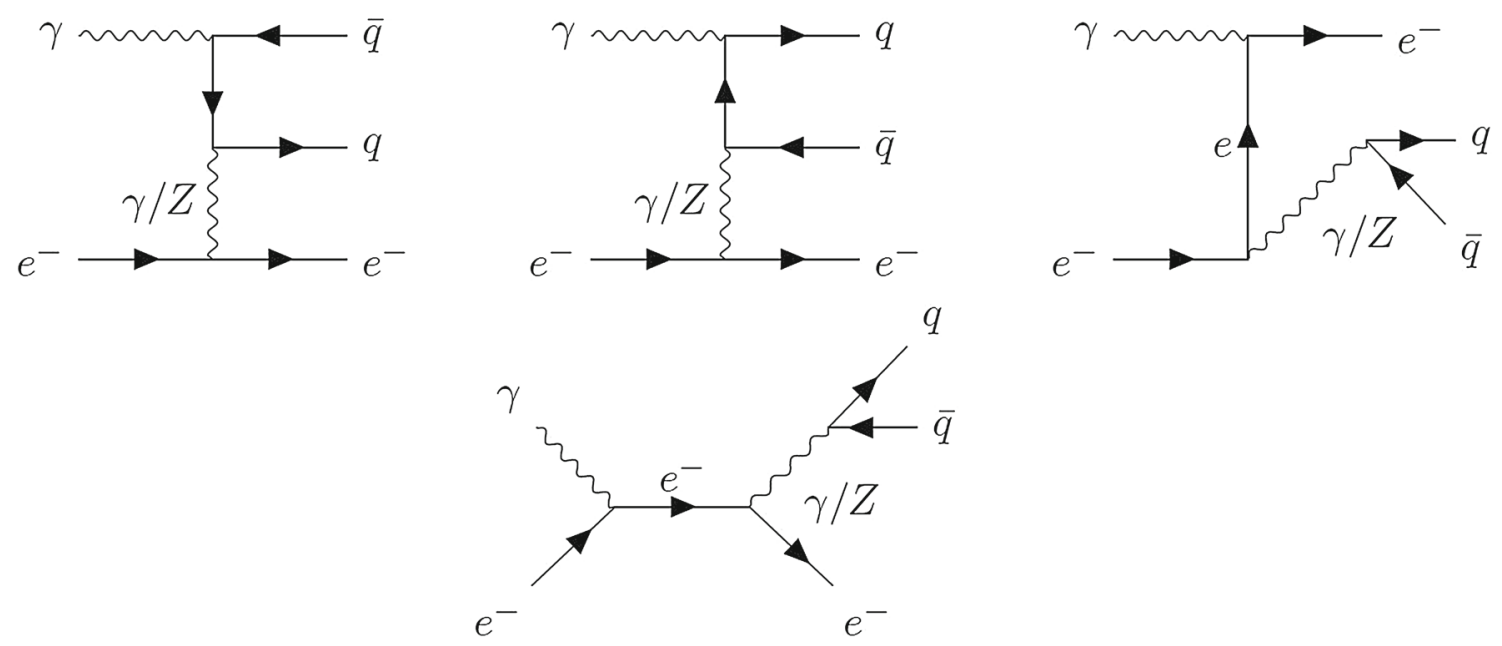

Fig. 4 Feynman diagrams for the SM background of the process $e^{-} \gamma \rightarrow e^{-}+2$ jets

- No jet should be accompanied by a hard lepton in the event.

- Jet-lepton isolation $\Delta R_{l j}>0.4$ and lepton-lepton isolation $\Delta R_{l l}>0.2$.

- Selected leptons are hadronically clean, i.e., hadronic activity within a cone of $\Delta R<0.3$ around each lepton should be less than $15 \%$ of the leptonic transverse momentum, i.e., $p_{T}^{\text {had }}<0.15 p_{T}^{\text {lep }}$ within the cone.

Prepared with this set up, we analyze different leptoquark scenarios and plot the required invariant masses for jet and lepton and their angular correlations. This will guide us to choose the kinematical cuts appropriately.

The leptoquark will eventually decay into a quark (or antiquark) and a lepton providing a mono-lepton plus di-jets signal at the electron photon collider. The SM background for this process, shown in Fig. 4, is governed by eight Feynman diagrams for each generation of quark-antiquark pair mediated by a photon and a $Z$-boson (neglecting the one with Higgs boson propagator, since its coupling with an electron is very small). While plotting against the invariant mass of the lepton-jet pair $\left(M_{\ell j}\right)$, the background gives a continuum, whereas the signal shows a peak at $M_{\phi}$. So, to reconstruct the leptoquark, we first put a cut constraining $\left(M_{\ell j}\right)$ to deviate from $M_{\phi}$ by $10 \mathrm{GeV}$ at most, which is denoted as "cut1" in all the signal-background analysis tables. Next, to distinguish the daughter jet produced by the decay of a leptoquark, we apply an angular cut on the angle between the lepton and each of the jets depending on the boost of the leptoquark. If the three-momentum of the leptoquark becomes small, the path of the daughter jet will make an obtuse angle with the final state lepton, providing negative values of $\cos \theta_{\ell j}$, whereas for a highly boosted leptoquark, it makes an acute angle with the lepton giving a positive valued $\cos \theta_{\ell j}$. To enhance the significance, we choose the angular cut in such a way that the background reduces conspicuously without much change in the signal event.

\subsection{Scalar leptoquarks}

\subsubsection{Leptoquark $\left(S^{+1 / 3}\right)^{c}$}

In Table 6, we summarize the signal-background analysis for the scalar leptoquark $\left(S_{1}^{+2 / 3}\right)^{c}$. In the case of BP1, all the three values of $\sqrt{s}$ (i.e. $200 \mathrm{GeV}, 2 \mathrm{TeV}$ and $3 \mathrm{TeV}$ ) are allowed for the production of $70 \mathrm{GeV}$ leptoquark associated with a light jet. As discussed in the previous paragraph, the leptoquark produced at $\sqrt{s}=200 \mathrm{GeV}$ will not be boosted highly, and 
Table 6 Signal-background analysis for leptoquark $\left(S^{+1 / 3}\right)^{c}$ with luminosity $100 \mathrm{fb}^{-1}$ at $e-\gamma$ collider

\begin{tabular}{|c|c|c|c|c|c|}
\hline Bench-mark points & $\sqrt{s}$ in $\mathrm{TeV}$ & Cut & Signal & Back-ground & Signi-ficance \\
\hline \multirow[t]{6}{*}{ BP1 } & \multirow[t]{2}{*}{0.2} & $\left|M_{l j}-M_{\phi}\right| \leq 10 \mathrm{GeV}$ & 11133.6 & 43725.0 & 47.5 \\
\hline & & $\operatorname{cut} 1+(-0.2) \leq \cos \theta_{\ell j} \leq 1$ & 10537.8 & 32989.8 & 50.5 \\
\hline & \multirow[t]{2}{*}{2} & $\left|M_{l j}-M_{\phi}\right| \leq 10 \mathrm{GeV}$ & 147.5 & 319.4 & 6.8 \\
\hline & & cut $1+(0.9) \leq \cos \theta_{\ell j} \leq 1$ & 91.5 & 114.2 & 6.4 \\
\hline & \multirow[t]{2}{*}{3} & $\left|M_{l j}-M_{\phi}\right| \leq 10 \mathrm{GeV}$ & 61.2 & 219.8 & 3.7 \\
\hline & & cut $1+(0.9) \leq \cos \theta_{\ell j} \leq 1$ & 34.5 & 44.2 & 3.9 \\
\hline \multirow[t]{4}{*}{$\mathrm{BP} 2$} & \multirow[t]{2}{*}{2} & $\left|M_{l j}-M_{\phi}\right| \leq 10 \mathrm{GeV}$ & 394.4 & 2003.6 & 8.1 \\
\hline & & cut $1+0 \leq \cos \theta_{\ell j} \leq 1$ & 299.5 & 129.1 & 14.5 \\
\hline & \multirow[t]{2}{*}{3} & $\left|M_{l j}-M_{\phi}\right| \leq 10 \mathrm{GeV}$ & 176.5 & 1660.7 & 4.1 \\
\hline & & cut $1+0 \leq \cos \theta_{\ell j} \leq 1$ & 159.0 & 167.5 & 8.8 \\
\hline \multirow[t]{4}{*}{ BP3 } & \multirow[t]{2}{*}{2} & $\left|M_{l j}-M_{\phi}\right| \leq 10 \mathrm{GeV}$ & 280.8 & 1061.6 & 7.7 \\
\hline & & cut $1+(-0.9) \leq \cos \theta_{\ell j} \leq 1$ & 199.8 & 391.5 & 8.2 \\
\hline & \multirow[t]{2}{*}{3} & $\left|M_{l j}-M_{\phi}\right| \leq 10 \mathrm{GeV}$ & 106.2 & 815.0 & 3.5 \\
\hline & & $\operatorname{cut} 1+(-0.8) \leq \cos \theta_{\ell j} \leq 1$ & 101.6 & 254.7 & 5.4 \\
\hline
\end{tabular}

hence we apply the angular cut as $-0.2 \leq \cos \theta_{\ell j} \leq 1$, which increases the significance from $47.5 \sigma$ to $50.5 \sigma$. But for $\sqrt{s}$ equal to $2 \mathrm{TeV}$ and $3 \mathrm{TeV}$ the leptoquark will be very highly boosted; therefore we put an angular cut of $0.9 \leq \cos \theta_{\ell j} \leq 1$, which changes the significance from $6.8 \sigma$ to $6.4 \sigma$ and $3.7 \sigma$ to $3.9 \sigma$, respectively. In the case of $\mathrm{BP} 2$, a center of momentum energy of $200 \mathrm{GeV}$ is forbidden for the production of a 650 $\mathrm{GeV}$ leptoquark. For the remaining two values of $\sqrt{s}$, the leptoquark will be moderately boosted. So, an angular cut of $0 \leq \cos \theta_{\ell j} \leq 1$ has been employed for the two cases. It elevates the significance from $8.1 \sigma$ to $14.5 \sigma$ and $4.1 \sigma$ to $8.8 \sigma$ for $\sqrt{s}$ of $2 \mathrm{TeV}$ and $3 \mathrm{TeV}$, respectively. On the other hand, for BP3 also, a real leptoquark gets produced at $2 \mathrm{TeV}$ and $3 \mathrm{TeV}$ center of momentum energy. At $\sqrt{s}=2 \mathrm{TeV}$, the produced leptoquark of mass $1.5 \mathrm{TeV}$ moves very slowly and hence an angular cut of $-0.9 \leq \cos \theta_{\ell j} \leq 1$ has been implemented, which enhances the significance to $7.7 \sigma$ from $8.2 \sigma$. Similarly, at $\sqrt{s}=3 \mathrm{TeV}$, also a slow leptoquark gets produced for BP3. So we put an angular cut of $-0.8 \leq$ $\cos \theta_{\ell j} \leq 1$, which enhances the significance to $5.4 \sigma$ from $3.5 \sigma$.

In Fig. 5, we present a detailed pictorial description of our PYTHIA simulation with $10^{5}$ events and a luminosity of $100 \mathrm{fb}^{-1}$. The graphs are arranged in the same order as in Table 6. In the left panel, the number of events has been plotted against the invariant mass of electron and jet for both signal and background at different center of mass energies for the three benchmark points. The greenish (aqua) regions indicate the SM background, whereas the purple regions signify the signal events. As expected, the signal events peak around the masses of leptoquarks. On the other hand, the number of events against the cosine of angle between the final state electron and the two jets has been plotted in the right panel for the same benchmark points with the same $\sqrt{s}$. While the blue and green lines represent the background events, the yellow and red lines depict the signal events. These plots justify our choice of cuts for the invariant mass and the angle between final state lepton and the two jets. If any of the two jets passes those two cuts, we identify that as a signal event.

In Fig. 6, the differential cross section is presented against the cosine of the angle between the initial state electron and the leptoquark (or equivalently, the angle between photon and the quark that is produced associated with the leptoquark) at different center of momentum energies for various benchmark points. The green (ragged) lines portray the simulated data with hundred bins within the range $-1<\cos \theta<1$, whereas the brown (smooth) lines represent the theoretical predictions given by Eq. (2.9). The plots are arranged in the order of Table 6 . The left and right plots at the top in $\mathrm{BP} 1$ row are for $200 \mathrm{GeV}$ and $2 \mathrm{TeV}$ center of momentum energies, respectively, while the third one is for $3 \mathrm{TeV}$. In the BP2 row, the first and second plots are done for the $2 \mathrm{TeV}$ and $3 \mathrm{TeV}$ center of momentum energies, respectively. Likewise, for BP3 also the plots for $\sqrt{s}$ valued 2 $\mathrm{TeV}$ and $3 \mathrm{TeV}$ are presented in the left and right panel of the third row. As can be seen, the angular distribution in each graph vanishes at some point, except the first one in the third row, which fails to satisfy the condition described by Eq. (2.12). The positions of the zeros can be verified from the left column (titled " $Q_{\bar{q}}=-2 / 3$ or $Q_{\phi}=-1 / 3$ ") of Table 5. 

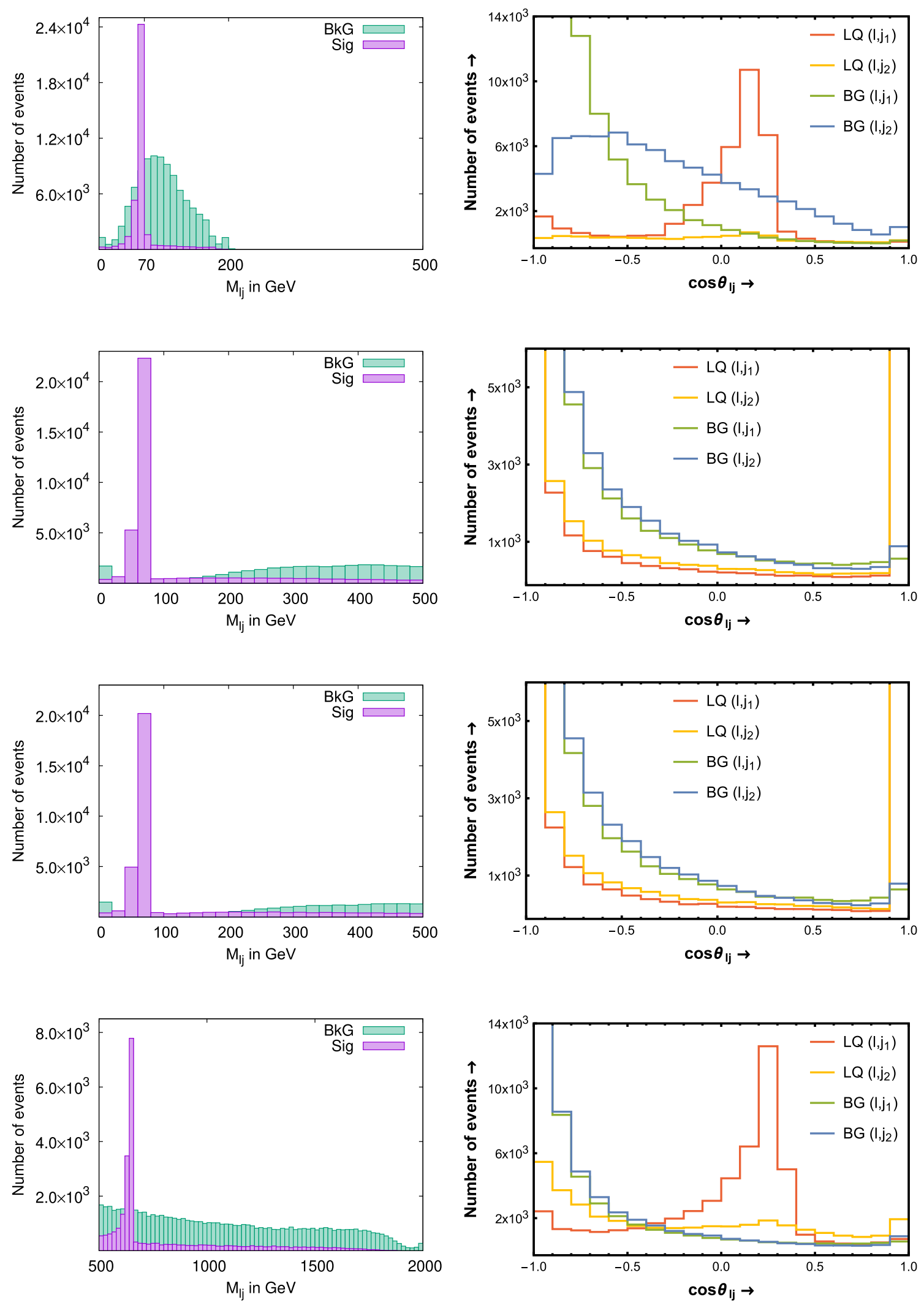

Fig. 5 Signal-background simulation for leptoquark $\left(S^{+1 / 3}\right)^{c}$ for $10^{5}$ number of events. The plots are organized in the same order as in Table 6 . In the left panel, we show the number of events against the invariant mass of the electron-jet pair for both signal (purple) and background (aqua). In the right panel we present the number of events for signal

and backgrounds against the cosine of the angle between the final state electron and the jet. The red and yellow symbolize the signal events for electron with first and second jet, respectively, whereas green and blue indicate the background events for the same 

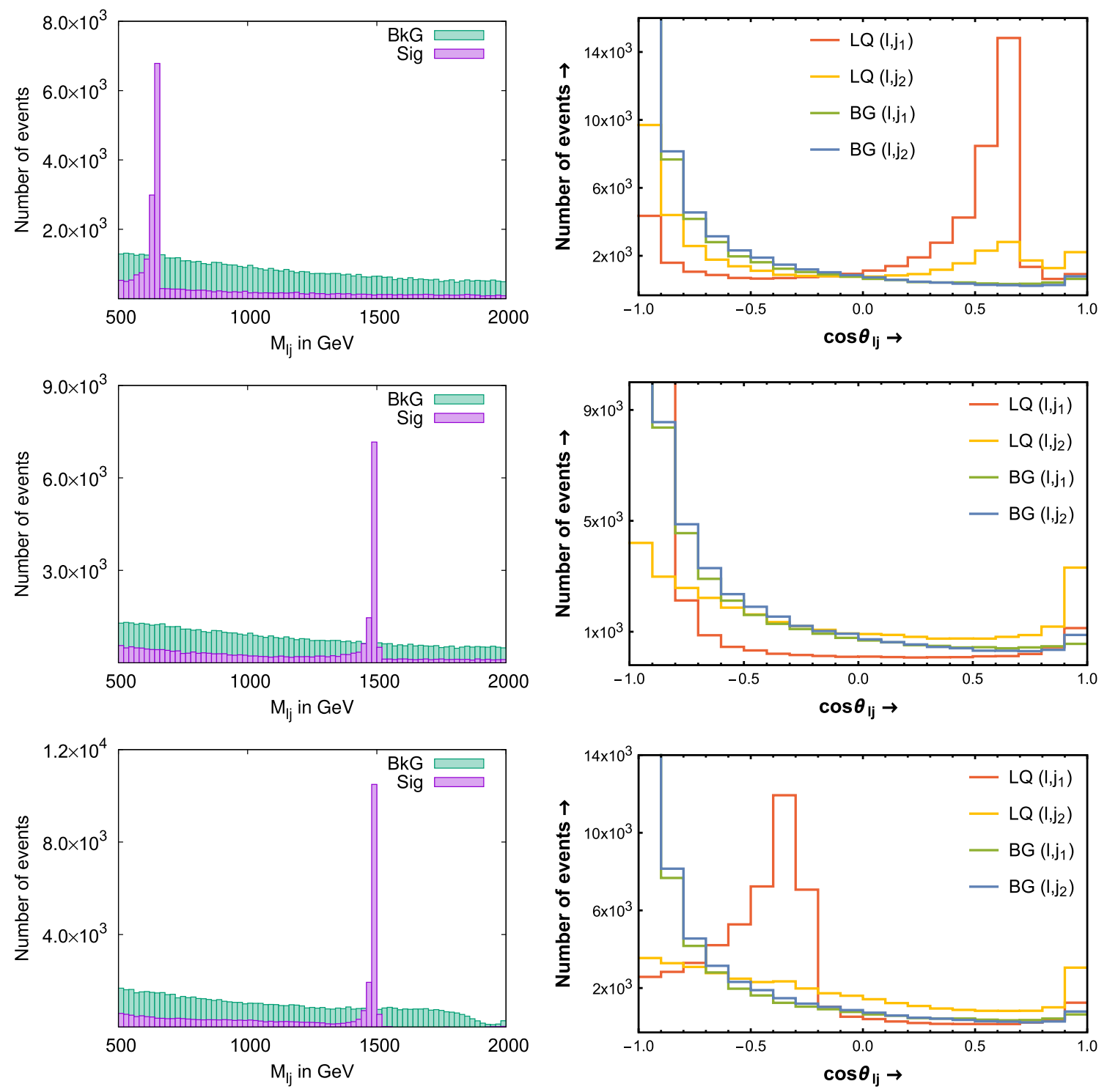

Fig. 5 continued 

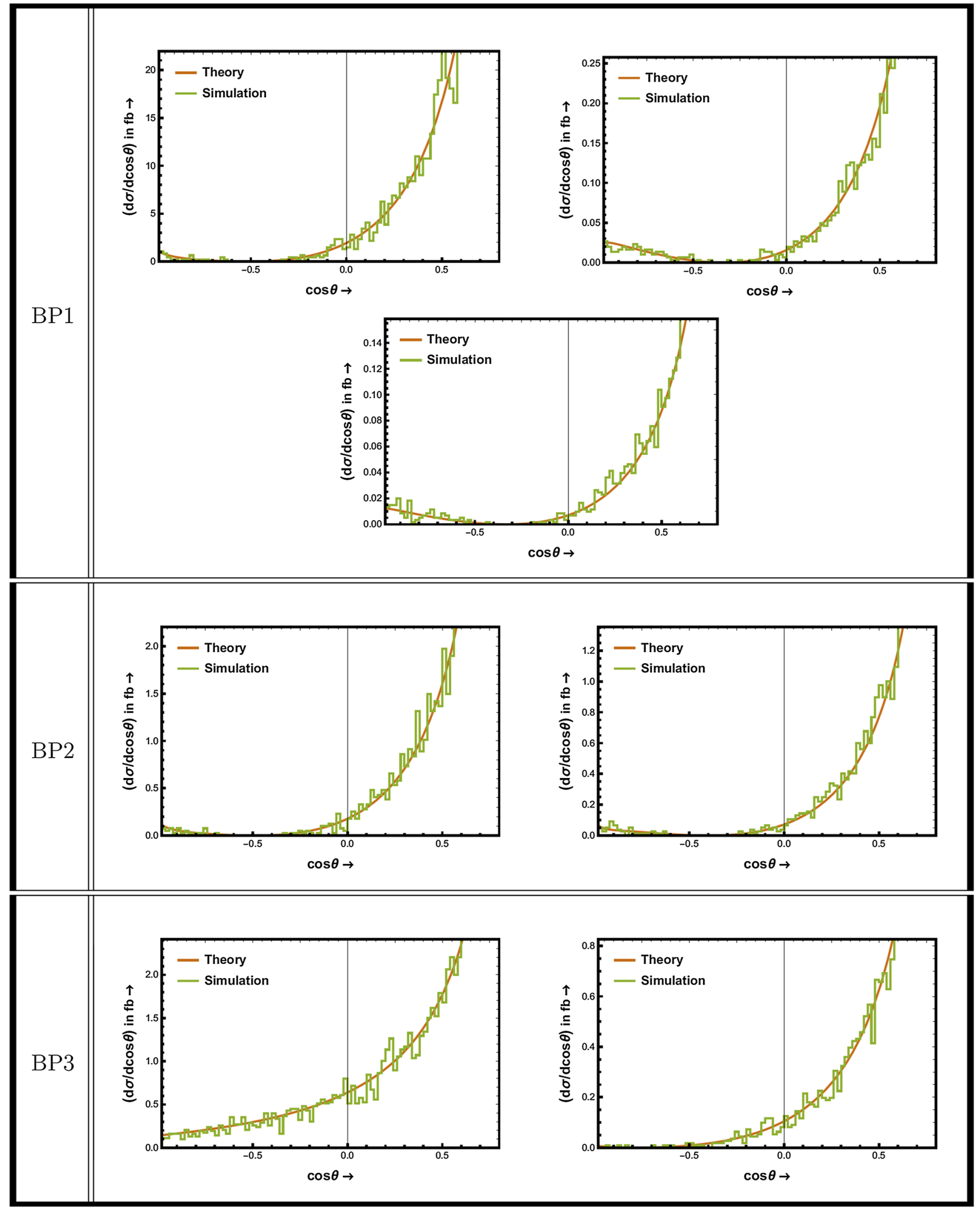

Fig. 6 Angular distribution for the production of $\left(S^{+1 / 3}\right)^{c}$ at various center of momentum energies for different benchmark points, arranged in the order of Table 6 . The brown (smooth) curves indicate the theoret- ical expectations, whereas the green (jagged) lines signify the PYTHIA simulated data 


\subsubsection{Leptoquark $\left(\widetilde{R}_{2}^{+2 / 3}\right)^{c}$}

The signal-background analysis for $\left(\widetilde{R}_{2}^{+2 / 3}\right)^{c}$ with luminosity of $100 \mathrm{fb}^{-1}$ is presented in Table 7 . For BP1, the cut on the invariant mass of the lepton-jet pair shows significances of $26.4 \sigma, 4.0 \sigma$ and $2.1 \sigma$ respectively, at three different values of the center of momentum energy; after applying the angular cuts, as described in the case of $\left(S_{1}^{+1 /}\right)^{c}$, the significances become $28.3 \sigma, 4.0 \sigma$ and $2.4 \sigma$, respectively. In the case of BP2, only significances of $0.6 \sigma$ and $0.3 \sigma$ are achieved by cut 1 at $2 \mathrm{TeV}$ and $3 \mathrm{TeV}$ center of momentum energies, respectively, which increase to $1.7 \sigma$ and $0.8 \sigma$, respectively, after implementation of the angular cut $0 \leq \cos \theta_{\ell j} \leq 1$. For BP3 with $2 \mathrm{TeV}$ energy, the significances reached by the two cuts are $0.6 \sigma$ and $0.7 \sigma$ and the same for $3 \mathrm{TeV}$ energy are $0.3 \sigma$ and $0.4 \sigma$, respectively. It should be noticed that the significances are quite low in the case of $\left(\widetilde{R}_{2}^{+2 / 3}\right)^{c}$ compared to $\left(S_{1}^{+1 /}\right)^{c}$, especially with BP2 and BP3, and hence escalation in luminosity is essential for amelioration of the statistics.

Angular distributions for this case are presented in Fig. 7 where the brown (even) and green (uneven) lines signify the theoretical estimates and simulated data, respectively. The plots are arranged in the same order as in Table 7. It can be observed that the distribution in every graph approaches zero at some point of phase space. The positions of the zeros can be verified from the right column (titled " $Q_{q}=-1 / 3$ or $Q_{\phi}=-2 / 3$ ") of Table 5 .

Table 7 Signal-background analysis for leptoquark $\left(\widetilde{R}_{2}^{+2 / 3}\right)^{c}$ with luminosity $100 \mathrm{fb}^{-1}$ at $e-\gamma$ collider

\begin{tabular}{|c|c|c|c|c|c|}
\hline Bench-mark points & $\sqrt{s}$ in $\mathrm{TeV}$ & Cut & Signal & Background & Significance \\
\hline \multirow[t]{6}{*}{ BP1 } & \multirow[t]{2}{*}{0.2} & $\left|M_{l j}-M_{\phi}\right| \leq 10 \mathrm{GeV}$ & 5870.1 & 43725.0 & 26.4 \\
\hline & & cut $1+(-0.2) \leq \cos \theta_{\ell j} \leq 1$ & 5549.6 & 32989.8 & 28.3 \\
\hline & \multirow[t]{2}{*}{2} & $\left|M_{l j}-M_{\phi}\right| \leq 10 \mathrm{GeV}$ & 80.3 & 319.4 & 4.0 \\
\hline & & $\operatorname{cut} 1+(0.9) \leq \cos \theta_{\ell j} \leq 1$ & 50.9 & 114.2 & 4.0 \\
\hline & \multirow[t]{2}{*}{3} & $\left|M_{l j}-M_{\phi}\right| \leq 10 \mathrm{GeV}$ & 33.6 & 219.8 & 2.1 \\
\hline & & $\operatorname{cut} 1+(0.9) \leq \cos \theta_{\ell j} \leq 1$ & 19.4 & 44.2 & 2.4 \\
\hline \multirow[t]{4}{*}{ BP2 } & \multirow[t]{2}{*}{2} & $\left|M_{l j}-M_{\phi}\right| \leq 10 \mathrm{GeV}$ & 27.0 & 2003.6 & 0.6 \\
\hline & & cut $1+0 \leq \cos \theta_{\ell j} \leq 1$ & 20.8 & 129.1 & 1.7 \\
\hline & \multirow[t]{2}{*}{3} & $\left|M_{l j}-M_{\phi}\right| \leq 10 \mathrm{GeV}$ & 11.99 & 1660.7 & 0.3 \\
\hline & & cut $1+0 \leq \cos \theta_{\ell j} \leq 1$ & 10.8 & 167.5 & 0.8 \\
\hline \multirow[t]{4}{*}{ BP3 } & \multirow[t]{2}{*}{2} & $\left|M_{l j}-M_{\phi}\right| \leq 10 \mathrm{GeV}$ & 19.4 & 1061.6 & 0.6 \\
\hline & & cut $1+(-0.9) \leq \cos \theta_{\ell j} \leq 1$ & 13.8 & 391.5 & 0.7 \\
\hline & \multirow[t]{2}{*}{3} & $\left|M_{l j}-M_{\phi}\right| \leq 10 \mathrm{GeV}$ & 7.6 & 815.0 & 0.3 \\
\hline & & $\operatorname{cut} 1+(-0.8) \leq \cos \theta_{\ell j} \leq 1$ & 7.2 & 254.7 & 0.4 \\
\hline
\end{tabular}




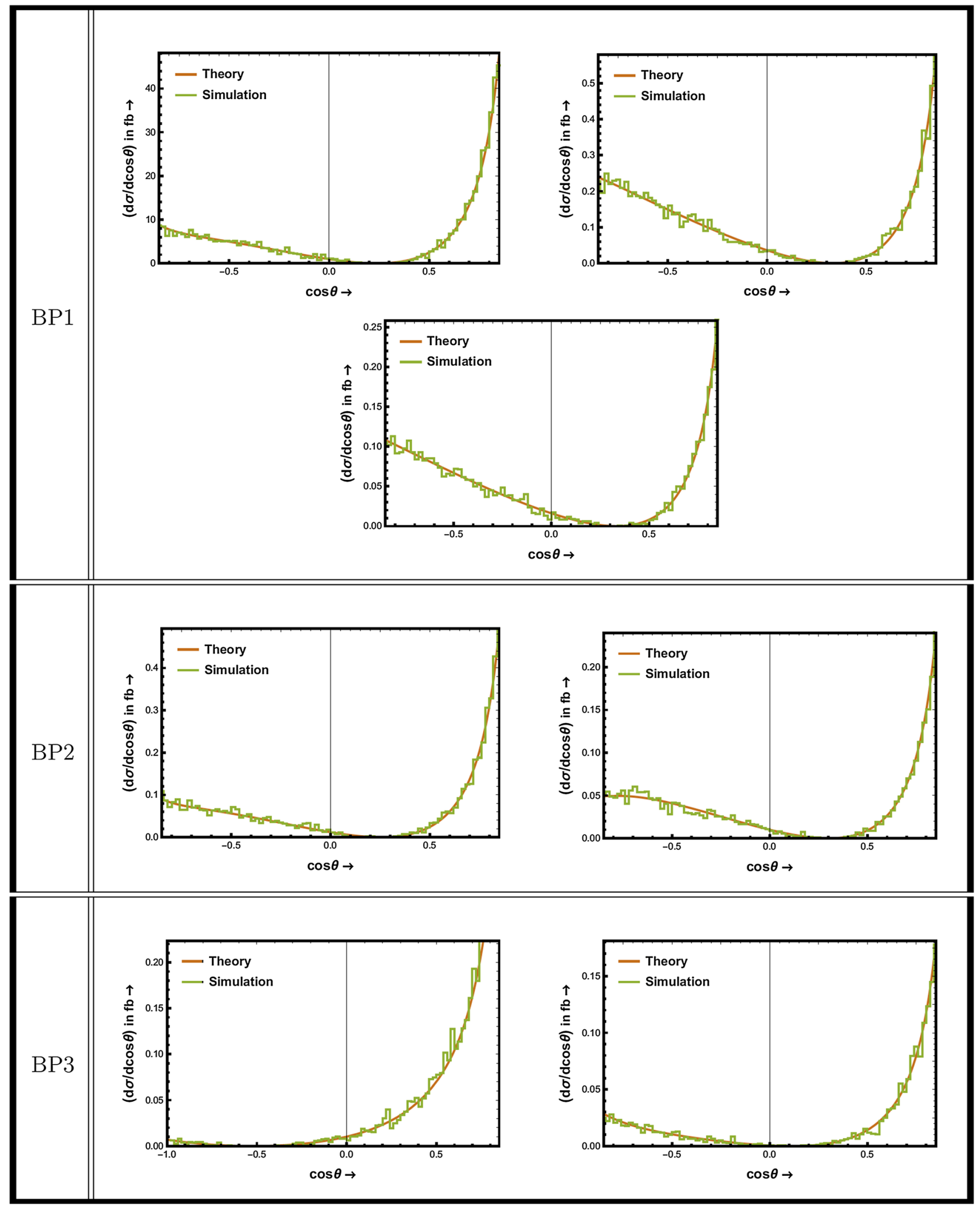

Fig. 7 Angular distribution for the production of $\left(\widetilde{R}_{2}^{+2 / 3}\right)^{c}$ at various center of momentum energies for different benchmark points, arranged in the order of Table 7. The brown (smooth) curves indicate the theoret- ical expectations, whereas the green (jagged) lines signify the PYTHIA simulated data 


\subsubsection{Leptoquark $\left(R_{2}^{+5 / 3}\right)^{c}$}

The PYTHIA analysis for the leptoquark $\left(R_{2}^{+5 / 3}\right)^{c}$ is presented in Table 8 . The cut on $M_{\ell j}$ provides significances of $94.5 \sigma, 13.9 \sigma$ and $7.8 \sigma$ for the signal events at three center of momentum energies in the case of BP1 which change to $98.7 \sigma, 13.2 \sigma$ and $8.1 \sigma$, respectively, after using suitable angular cuts on $\cos \theta_{\ell j}$. For BP2, signal events are produced with significances $14.4 \sigma$ and $8.1 \sigma$ at $2 \mathrm{TeV}$ and 3 $\mathrm{TeV}$ center of momentum energies, respectively, any they get increased to $21.9 \sigma$ and $14.8 \sigma$ after applying the angular cut as $0 \leq \cos \theta_{\ell j} \leq 1$. For BP3 at $\sqrt{s}=2 \mathrm{TeV}$, the significances become $11.4 \sigma$ and $11.7 \sigma$ after implementation of the two cuts and the same become $5.9 \sigma$ and $8.6 \sigma$, respectively, for $\sqrt{s}=3 \mathrm{TeV}$.

Figure 8 describes the differential distribution with respect to the cosine of the angle between leptoquark and initial state electron in this scenario. The plots are arranged in the order of Table 8. As before the green (jagged) and brown (smooth) lines indicate the simulated data with 100 bins and the theoretical expectation for various benchmark points at different center of momentum energy, respectively. Unlike the other two cases, the angular distributions never vanish inside the physical region, since this leptoquark does not satisfy Eq. (2.13).

Table 8 Signal-background analysis for leptoquark $\left(R_{2}^{+5 / 3}\right)^{c}$ with luminosity $100 \mathrm{fb}^{-1}$ at $e-\gamma$ collider

\begin{tabular}{|c|c|c|c|c|c|}
\hline Bench-mark points & $\sqrt{s}$ in $\mathrm{TeV}$ & Cut & Signal & Back-ground & Signi-ficance \\
\hline \multirow[t]{6}{*}{ BP1 } & \multirow[t]{2}{*}{0.2} & $\left|M_{l j}-M_{\phi}\right| \leq 10 \mathrm{GeV}$ & 24719.2 & 43725.0 & 94.5 \\
\hline & & $\operatorname{cut} 1+(-0.2) \leq \cos \theta_{\ell j} \leq 1$ & 23448.8 & 32989.8 & 98.7 \\
\hline & \multirow[t]{2}{*}{2} & $\left|M_{l j}-M_{\phi}\right| \leq 10 \mathrm{GeV}$ & 365.7 & 319.4 & 13.9 \\
\hline & & $\operatorname{cut} 1+(0.9) \leq \cos \theta_{\ell j} \leq 1$ & 251.7 & 114.2 & 13.2 \\
\hline & \multirow[t]{2}{*}{3} & $\left|M_{l j}-M_{\phi}\right| \leq 10 \mathrm{GeV}$ & 148.9 & 219.8 & 7.8 \\
\hline & & $\operatorname{cut} 1+(0.9) \leq \cos \theta_{\ell j} \leq 1$ & 96.2 & 44.2 & 8.1 \\
\hline \multirow[t]{4}{*}{ BP2 } & \multirow[t]{2}{*}{2} & $\left|M_{l j}-M_{\phi}\right| \leq 10 \mathrm{GeV}$ & 757.4 & 2003.6 & 14.4 \\
\hline & & cut $1+0 \leq \cos \theta_{\ell j} \leq 1$ & 585.5 & 129.1 & 21.9 \\
\hline & \multirow[t]{2}{*}{3} & $\left|M_{l j}-M_{\phi}\right| \leq 10 \mathrm{GeV}$ & 362.6 & 1660.7 & 8.1 \\
\hline & & cut $1+0 \leq \cos \theta_{\ell j} \leq 1$ & 329.5 & 167.5 & 14.8 \\
\hline \multirow[t]{4}{*}{ BP3 } & \multirow[t]{2}{*}{2} & $\left|M_{l j}-M_{\phi}\right| \leq 10 \mathrm{GeV}$ & 440.9 & 1061.6 & 11.4 \\
\hline & & $\operatorname{cut} 1+(-0.9) \leq \cos \theta_{\ell j} \leq 1$ & 311.2 & 391.5 & 11.7 \\
\hline & \multirow[t]{2}{*}{3} & $\left|M_{l j}-M_{\phi}\right| \leq 10 \mathrm{GeV}$ & 187.8 & 815.0 & 5.9 \\
\hline & & cut $1+(-0.8) \leq \cos \theta_{\ell j} \leq 1$ & 180.0 & 254.7 & 8.6 \\
\hline
\end{tabular}




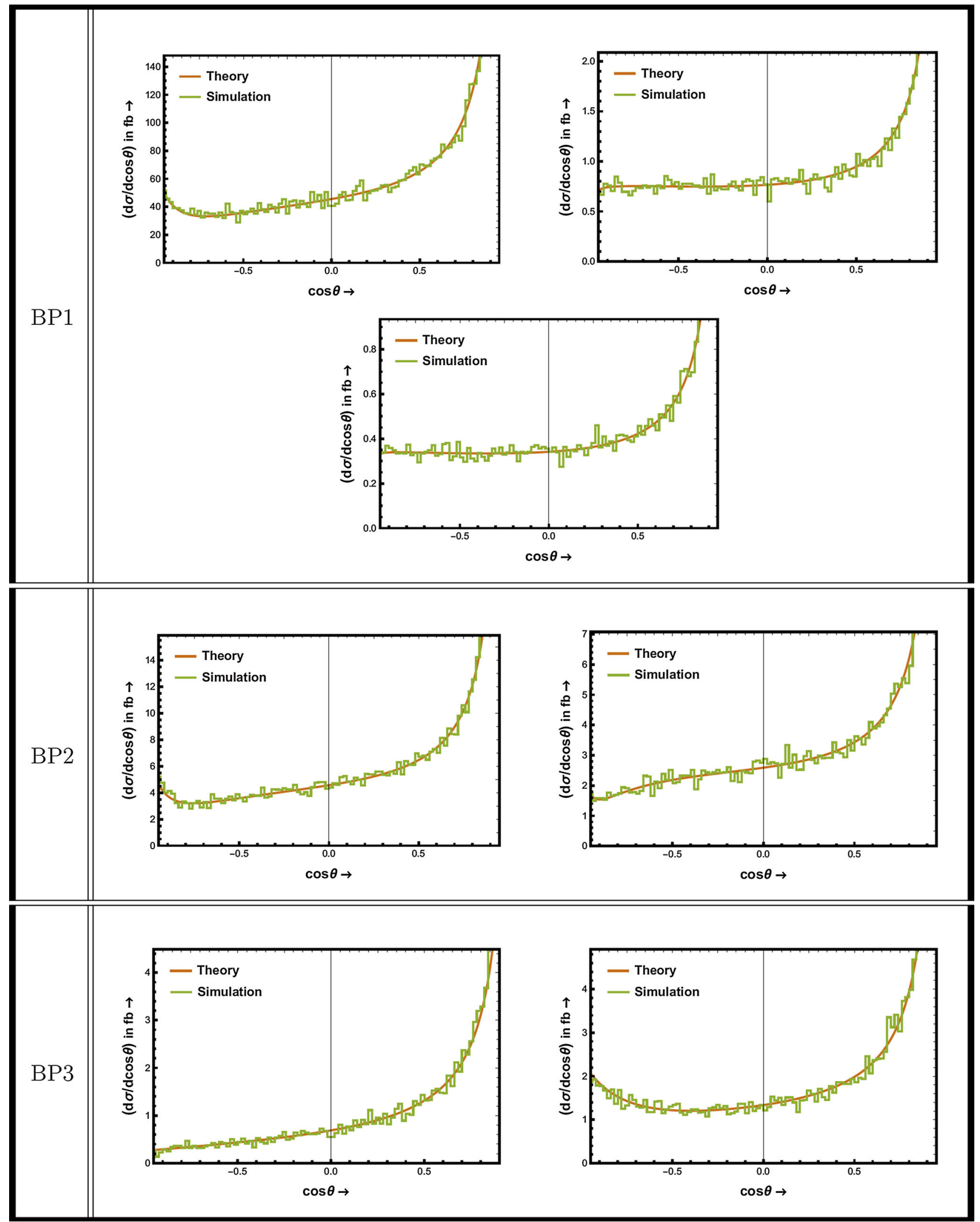

Fig. 8 Angular distribution for the production of $\left(R_{2}^{+5 / 3}\right)^{c}$ at various center of momentum energies for different benchmark points, arranged in the order of Table 8 . The brown (smooth) curves indicate the theoret- ical expectations, whereas the green (jagged) lines signify the PYTHIA simulated data 


\subsubsection{Leptoquarks $\left(S_{3}^{+4 / 3}\right)^{c}$}

The signal-background analysis for $\left(\widetilde{S}_{3}^{+4 / 3}\right)^{c}$ with a luminosity of $100 \mathrm{fb}^{-1}$ is presented in Table 9. For BP1, the cut on the invariant mass of the lepton-jet pair shows significances of $36.1 \sigma, 6.2 \sigma$ and $3.2 \sigma$ at center of momentum energies of $200 \mathrm{GeV}, 2 \mathrm{TeV}$ and $3 \mathrm{TeV}$ respectively. The angular cuts modify these significances to become $38.7 \sigma$, $6.8 \sigma$ and $4.2 \sigma$, respectively. In the case of BP2, the significances achieved by cut 1 at $2 \mathrm{TeV}$ and $3 \mathrm{TeV}$ center of momentum energies are $0.9 \sigma$ and $0.5 \sigma$ only, which increase to $2.5 \sigma$ and $1.4 \sigma$, respectively, after implementation of the angular cut $0 \leq \cos \theta_{\ell j} \leq 1$. For BP3 with $2 \mathrm{TeV}$ energy, the significances reached by the two cuts are $0.6 \sigma$ and $0.7 \sigma$, respectively, which change to $0.3 \sigma$ and $0.7 \sigma$ at $\sqrt{s}$ to be 3 $\mathrm{TeV}$. In this case also the significances are quite low compared to $\left(S_{1}^{+1 / 3}\right)^{c}$, especially with BP2 and BP3. An increase in luminosity is needed for improvement of the statistics.

Figure 9 shows the comparison between theoretical expectation and PYTHIA simulated data for the production of $\left(S_{3}^{+4 / 3}\right)^{c}$. The plots are arranged in the order of Table 9. As before the green (uneven) and brown (even) lines indicate the simulated data with 100 bins and the theoretical expectation for various benchmark points at different center of momentum energies, respectively. In this case also no zero of differential distribution in any of the diagrams is found since its charge is smaller than -1 unit.

Table 9 Signal-background analysis for leptoquark $\left(S_{3}^{+4 / 3}\right)^{c}$ with luminosity $100 \mathrm{fb}^{-1}$ at $e-\gamma$ collider

\begin{tabular}{|c|c|c|c|c|c|}
\hline Bench-mark points & $\sqrt{s}$ in $\mathrm{TeV}$ & Cut & Signal & Back-ground & Signi-ficance \\
\hline \multirow[t]{6}{*}{ BP1 } & \multirow[t]{2}{*}{0.2} & $\left|M_{l j}-M_{\phi}\right| \leq 10 \mathrm{GeV}$ & 8237.3 & 43725.0 & 36.1 \\
\hline & & $\operatorname{cut} 1+(-0.2) \leq \cos \theta_{\ell j} \leq 1$ & 7812.9 & 32989.8 & 38.7 \\
\hline & \multirow[t]{2}{*}{2} & $\left|M_{l j}-M_{\phi}\right| \leq 10 \mathrm{GeV}$ & 132.7 & 319.4 & 6.2 \\
\hline & & $\operatorname{cut} 1+(0.9) \leq \cos \theta_{\ell j} \leq 1$ & 99.3 & 114.2 & 6.8 \\
\hline & \multirow[t]{2}{*}{3} & $\left|M_{l j}-M_{\phi}\right| \leq 10 \mathrm{GeV}$ & 53.6 & 219.8 & 3.2 \\
\hline & & cut $1+(0.9) \leq \cos \theta_{\ell j} \leq 1$ & 38.0 & 44.2 & 4.2 \\
\hline \multirow[t]{4}{*}{ BP2 } & \multirow[t]{2}{*}{2} & $\left|M_{l j}-M_{\phi}\right| \leq 10 \mathrm{GeV}$ & 40.4 & 2003.6 & 0.9 \\
\hline & & cut $1+0 \leq \cos \theta_{\ell j} \leq 1$ & 31.4 & 129.1 & 2.5 \\
\hline & \multirow[t]{2}{*}{3} & $\left|M_{l j}-M_{\phi}\right| \leq 10 \mathrm{GeV}$ & 21.2 & 1660.7 & 0.5 \\
\hline & & $\operatorname{cut} 1+0 \leq \cos \theta_{\ell j} \leq 1$ & 19.6 & 167.5 & 1.4 \\
\hline \multirow[t]{4}{*}{ BP3 } & \multirow[t]{2}{*}{2} & $\left|M_{l j}-M_{\phi}\right| \leq 10 \mathrm{GeV}$ & 20.4 & 1061.6 & 0.6 \\
\hline & & $\operatorname{cut} 1+(-0.9) \leq \cos \theta_{\ell j} \leq 1$ & 14.4 & 391.5 & 0.7 \\
\hline & \multirow[t]{2}{*}{3} & $\left|M_{l j}-M_{\phi}\right| \leq 10 \mathrm{GeV}$ & 9.7 & 815.0 & 0.3 \\
\hline & & cut $1+(-0.8) \leq \cos \theta_{\ell j} \leq 1$ & 9.3 & 254.7 & 0.6 \\
\hline
\end{tabular}




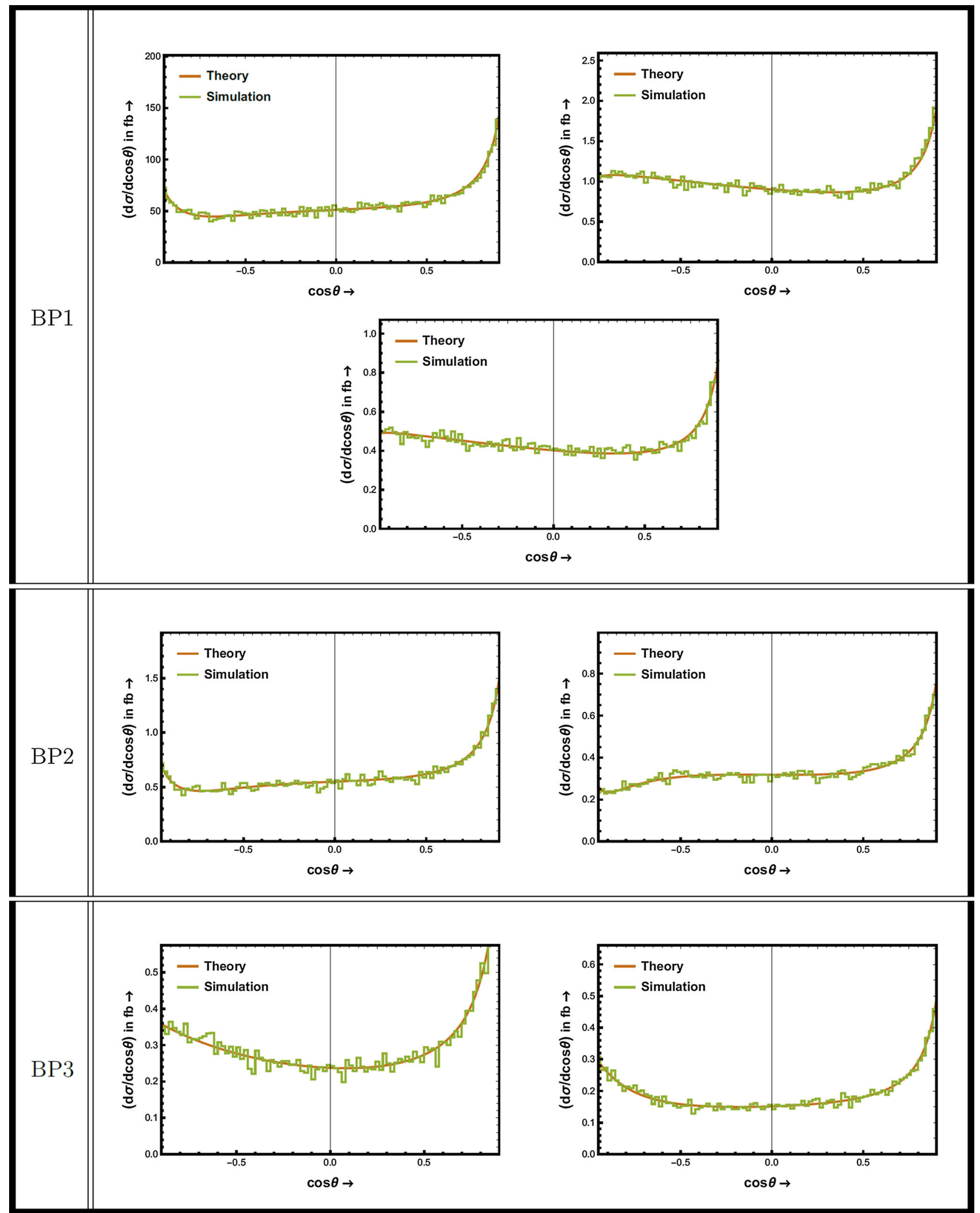

Fig. 9 Angular distribution for the production of $\left(S_{3}^{+4 / 3}\right)^{c}$ at various center of momentum energies for different benchmark points, arranged in the order of Table 9. The brown (smooth) curves indicate the theoret- ical expectations whereas the green (jagged) lines signify the PYTHIA simulated data 


\subsection{Vector leptoquarks}

\subsubsection{Leptoquark $\left(U_{1 \mu}^{+2 / 3}\right)^{c}$}

In Table 10, we summarize the signal-background analysis for the vector singlet leptoquark $\left(U_{1 \mu}^{+2 / 3}\right)^{c}$. For BP1 at $\sqrt{s}=200 \mathrm{GeV}$, the invariant mass cut of $10 \mathrm{GeV}$ gives $44.7 \sigma$ significance and further application of the angular cut of $(-0.2) \leq \cos \theta_{\ell j} \leq 1$ changes it to $46.9 \sigma$. For BP1 at center of momentum energies to be $2 \mathrm{TeV}$ and $3 \mathrm{TeV}$, the significances after the first cut are $119.8 \sigma$ and $120.8 \sigma$, respectively. In these cases, the signal events after the first cut are so large in number relative to the background events that the angular cut becomes obsolete. In the case of BP2, the cut on $M_{\ell j}$ pro- duce signal events with significances $9.0 \sigma$ and $11.3 \sigma$ for the two values of $\sqrt{s}$, which get enhanced to $15.6 \sigma$ and $19.0 \sigma$, respectively, after constraining the angle $\theta_{\ell j}$ within the limit $0 \leq \cos \theta_{\ell j} \leq 1$. For BP3 at $\sqrt{s}=2 \mathrm{TeV}$, the angular cut increases the significance to $4.6 \sigma$ from $4.2 \sigma$. Likewise, for $\mathrm{BP} 3$ at $\sqrt{s}=2 \mathrm{TeV}$, the angular cut increases the significance to $3.4 \sigma$ from $2.2 \sigma$.

Angular distribution for this case is presented in Fig. 10 where the brown (smooth) and green (ragged) lines signify the theoretical estimates and simulated data, respectively. The plots are arranged in terms of benchmark points and center of momentum energy according to Table 10. All the curves show zero certainly at some points of phase space which matches with the right column of Table 5 .

Table 10 Signal-background analysis for $\left(U_{1 \mu}^{+2 / 3}\right)^{c}$ with luminosity $100 \mathrm{fb}^{-1}$ at $e-\gamma$ collider

\begin{tabular}{|c|c|c|c|c|c|}
\hline Bench-mark points & $\sqrt{s}$ in $\mathrm{TeV}$ & Cut & Signal & Back-ground & Signi-ficance \\
\hline \multirow[t]{6}{*}{ BP1 } & \multirow[t]{2}{*}{0.2} & $\left|M_{l j}-M_{\phi}\right| \leq 10 \mathrm{GeV}$ & 10399.3 & 43725.0 & 44.7 \\
\hline & & cut $1+(-0.2) \leq \cos \theta_{\ell j} \leq 1$ & 9700.3 & 32989.8 & 46.9 \\
\hline & \multirow[t]{2}{*}{2} & $\left|M_{l j}-M_{\phi}\right| \leq 10 \mathrm{GeV}$ & 14666.5 & 319.4 & 119.8 \\
\hline & & $\operatorname{cut} 1+(0.9) \leq \cos \theta_{\ell j} \leq 1$ & 9555.0 & 114.2 & 97.17 \\
\hline & \multirow[t]{2}{*}{3} & $\left|M_{l j}-M_{\phi}\right| \leq 10 \mathrm{GeV}$ & 14799.6 & 219.8 & 120.8 \\
\hline & & $\operatorname{cut} 1+(0.9) \leq \cos \theta_{\ell j} \leq 1$ & 8736.1 & 44.2 & 93.2 \\
\hline \multirow[t]{4}{*}{ BP2 } & \multirow[t]{2}{*}{2} & $\left|M_{l j}-M_{\phi}\right| \leq 10 \mathrm{GeV}$ & 443.3 & 2003.6 & 9.0 \\
\hline & & cut $1+0 \leq \cos \theta_{\ell j} \leq 1$ & 337.5 & 129.1 & 15.6 \\
\hline & \multirow[t]{2}{*}{3} & $\left|M_{l j}-M_{\phi}\right| \leq 10 \mathrm{GeV}$ & 530.0 & 1660.7 & 11.3 \\
\hline & & cut $1+0 \leq \cos \theta_{\ell j} \leq 1$ & 483.7 & 167.5 & 19.0 \\
\hline \multirow[t]{4}{*}{ BP3 } & \multirow[t]{2}{*}{2} & $\left|M_{l j}-M_{\phi}\right| \leq 10 \mathrm{GeV}$ & 144.4 & 1061.6 & 4.2 \\
\hline & & $\operatorname{cut} 1+(-0.9) \leq \cos \theta_{\ell j} \leq 1$ & 102.2 & 391.5 & 4.6 \\
\hline & \multirow[t]{2}{*}{3} & $\left|M_{l j}-M_{\phi}\right| \leq 10 \mathrm{GeV}$ & 63.9 & 815.0 & 2.2 \\
\hline & & $\operatorname{cut} 1+(-0.8) \leq \cos \theta_{\ell j} \leq 1$ & 60.7 & 254.7 & 3.4 \\
\hline
\end{tabular}




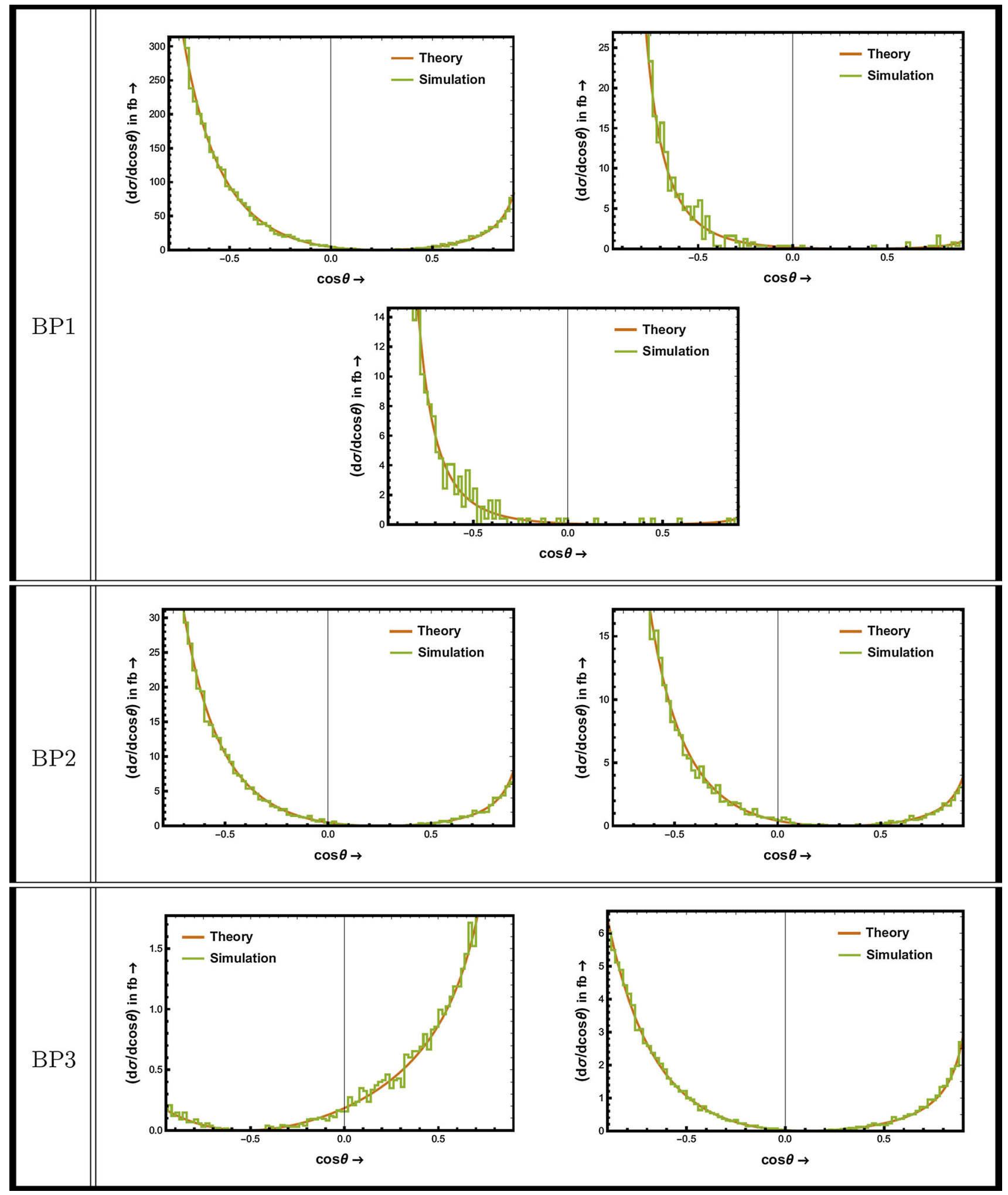

Fig. 10 Angular distribution for the production of $\left(U_{1 \mu}^{+2 / 3}\right)^{c}$ at various center of momentum energies for different benchmark points, arranged in the order of Table 10. The brown (smooth) and green (jagged) lines indicate the theoretical expectations and the PYTHIA simulated data 


\subsubsection{Leptoquark $\left(V_{2 \mu}^{+4 / 3}\right)^{c}$}

The signal-background analysis for leptoquark $\left(V_{2 \mu}^{+4 / 3}\right)^{c}$ is shown in Table 11. For BP1, the significances of leptoquark production is very high $(506.2 \sigma, 631.5 \sigma$ and $635.8 \sigma)$ at all the three values of $\sqrt{s}$ and angular cuts become almost obsolete. For BP2, the significances after the first cut are $26.2 \sigma$ and $32.1 \sigma$ which get enhanced to $33.1 \sigma$ and $40.0 \sigma$, respectively, after the second cut at the two different values of $\sqrt{s}$. For BP3 at $2 \mathrm{TeV}$ center of momentum energy the significances after the two cuts are $3.5 \sigma$ and $3.9 \sigma$, respectively. At
$3 \mathrm{TeV}$ center of momentum energy for the same benchmark point, the significances after the two cuts become $4.5 \sigma$ and $6.7 \sigma$, respectively.

In Fig. 11, we show the angular distribution for the production of leptoquark $\left(V_{2 \mu}^{+4 / 3}\right)^{c}$ associated with an antiquark $\bar{d}$ for all the three benchmark points at different center of momentum energies as described in Table 11. As before, the brown (even) and green (uneven) lines signify the theoretical expectations and the PYTHIA simulated data, respectively. In this case also, no zero in any of the plots is found.

Table 11 Signal-background analysis for $\left(V_{2 \mu}^{+4 / 3}\right)^{c}$ with luminosity $100 \mathrm{fb}^{-1}$ at $e-\gamma$ collider

\begin{tabular}{|c|c|c|c|c|c|}
\hline Bench-mark points & $\sqrt{s}$ in $\mathrm{TeV}$ & Cut & Signal & Back-ground & Signi-ficance \\
\hline \multirow[t]{6}{*}{ BP1 } & \multirow[t]{2}{*}{0.2} & $\left|M_{l j}-M_{\phi}\right| \leq 10 \mathrm{GeV}$ & 294306.3 & 43725.0 & 506.2 \\
\hline & & cut $1+(-0.2) \leq \cos \theta_{\ell j} \leq 1$ & 275902.1 & 32989.8 & 496.4 \\
\hline & \multirow[t]{2}{*}{2} & $\left|M_{l j}-M_{\phi}\right| \leq 10 \mathrm{GeV}$ & 399147.0 & 319.4 & 631.5 \\
\hline & & cut $1+(0.9) \leq \cos \theta_{\ell j} \leq 1$ & 257096.9 & 114.2 & 506.9 \\
\hline & \multirow[t]{2}{*}{3} & $\left|M_{l j}-M_{\phi}\right| \leq 10 \mathrm{GeV}$ & 404429.7 & 219.8 & 635.8 \\
\hline & & cut $1+(0.9) \leq \cos \theta_{\ell j} \leq 1$ & 238127.0 & 44.2 & 487.9 \\
\hline \multirow[t]{4}{*}{ BP2 } & \multirow[t]{2}{*}{2} & $\left|M_{l j}-M_{\phi}\right| \leq 10 \mathrm{GeV}$ & 1560.1 & 2003.6 & 26.1 \\
\hline & & $\operatorname{cut} 1+0 \leq \cos \theta_{\ell j} \leq 1$ & 1215.5 & 129.1 & 33.1 \\
\hline & \multirow[t]{2}{*}{3} & $\left|M_{l j}-M_{\phi}\right| \leq 10 \mathrm{GeV}$ & 1920.1 & 1660.7 & 32.1 \\
\hline & & cut $1+0 \leq \cos \theta_{\ell j} \leq 1$ & 1754.7 & 167.5 & 40.0 \\
\hline \multirow[t]{4}{*}{ BP3 } & \multirow[t]{2}{*}{2} & $\left|M_{l j}-M_{\phi}\right| \leq 10 \mathrm{GeV}$ & 119.3 & 1061.6 & 3.5 \\
\hline & & cut $1+(-0.9) \leq \cos \theta_{\ell j} \leq 1$ & 85.5 & 391.5 & 3.9 \\
\hline & \multirow[t]{2}{*}{3} & $\left|M_{l j}-M_{\phi}\right| \leq 10 \mathrm{GeV}$ & 139.1 & 815.0 & 4.5 \\
\hline & & $\operatorname{cut} 1+(-0.8) \leq \cos \theta_{\ell j} \leq 1$ & 132.4 & 254.7 & 6.7 \\
\hline
\end{tabular}




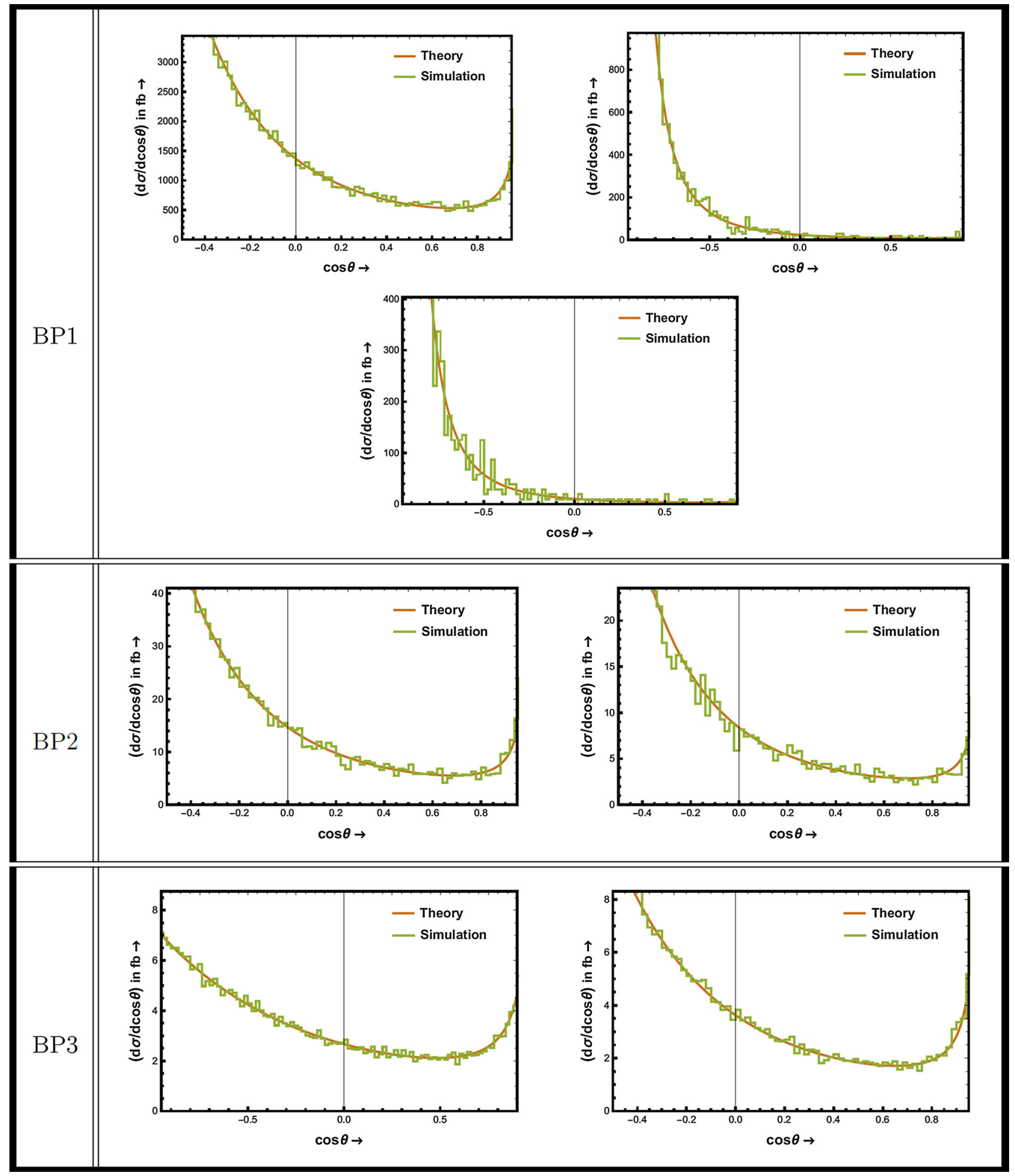

Fig. 11 Angular distribution for the production of $\left(V_{2 \mu}^{+4 / 3}\right)^{c}$ at various center of momentum energies for different benchmark points, arranged in the order of Table 11. The brown (smooth) and green (jagged) lines indicate the theoretical expectations the PYTHIA simulated data 


\subsubsection{Leptoquark $\left(\widetilde{V}_{2 \mu}^{+1 / 3}\right)^{c}$}

Table 12 summarizes the reconstruction of the leptoquark $\left(\widetilde{V}_{2 \mu}^{+1 / 3}\right)^{c}$ at $100 \mathrm{fb}^{-1}$ luminosity. In this case also the significance for production of the leptoquark is quite high after the first cut for BP1 $(267.4 \sigma, 130.6 \sigma$ and $128.8 \sigma$, respectively,) and hence the second cuts become unimportant. For $\mathrm{BP} 2$, the significances after the invariant mass cut are $5.0 \sigma$ and $2.8 \sigma$, which get improved to $10.2 \sigma$ and $6.4 \sigma$, respectively, after the angular cut for the $2 \mathrm{TeV}$ and $3 \mathrm{TeV}$ center of momentum energies, respectively. For BP3 at $\sqrt{s}=2 \mathrm{TeV}$, the significance goes to $4.9 \sigma$ from $4.4 \sigma$ after implementing the angular cut of $(-0.8) \leq \cos \theta_{\ell j} \leq 1$. For $\sqrt{s}=3 \mathrm{TeV}$ the corresponding change in significance is from $2.1 \sigma$ to $4.2 \sigma$.

In Fig. 12, we show the differential distribution for the production of this leptoquark. We ordered the graphs in the same way as in Table 12. The brown (smooth) and green (coarse) lines signify the theoretical estimates and the simulated data, respectively. As expected the distributions at different center of momentum energies for various benchmark points go to zero at different points of phase space except the plot at the left panel in the third row. The positions of the zeros can be verified from the left column (titled " $Q_{\bar{q}}=-2 / 3$ or $\left.Q_{\phi}=-1 / 3 "\right)$ of Table 5 .

Table 12 Signal-background analysis for leptoquark $\left(\widetilde{V}_{2 \mu}^{+1 / 3}\right)^{c}$ with luminosity $100 \mathrm{fb}^{-1}$ at $e-\gamma$ collider

\begin{tabular}{|c|c|c|c|c|c|}
\hline Bench-mark points & $\sqrt{s}$ in $\mathrm{TeV}$ & Cut & Signal & Back-ground & Signi-ficance \\
\hline \multirow[t]{6}{*}{ BP1 } & \multirow[t]{2}{*}{0.2} & $\left|M_{l j}-M_{\phi}\right| \leq 10 \mathrm{GeV}$ & 102107.7 & 43725.0 & 267.4 \\
\hline & & $\operatorname{cut} 1+(-0.2) \leq \cos \theta_{\ell j} \leq 1$ & 96573.2 & 32989.8 & 268.3 \\
\hline & \multirow[t]{2}{*}{2} & $\left|M_{l j}-M_{\phi}\right| \leq 10 \mathrm{GeV}$ & 17380.0 & 319.4 & 130.6 \\
\hline & & cut $1+(0.9) \leq \cos \theta_{\ell j} \leq 1$ & 11072.0 & 114.2 & 104.7 \\
\hline & \multirow[t]{2}{*}{3} & $\left|M_{l j}-M_{\phi}\right| \leq 10 \mathrm{GeV}$ & 16809.0 & 219.8 & 128.8 \\
\hline & & $\operatorname{cut} 1+(0.9) \leq \cos \theta_{\ell j} \leq 1$ & 9738.8 & 44.2 & 98.5 \\
\hline \multirow[t]{4}{*}{ BP2 } & \multirow[t]{2}{*}{2} & $\left|M_{l j}-M_{\phi}\right| \leq 10 \mathrm{GeV}$ & 236.5 & 2003.6 & 5.0 \\
\hline & & $\operatorname{cut} 1+0 \leq \cos \theta_{\ell j} \leq 1$ & 179.6 & 129.1 & 10.2 \\
\hline & \multirow[t]{2}{*}{3} & $\left|M_{l j}-M_{\phi}\right| \leq 10 \mathrm{GeV}$ & 117.5 & 1660.7 & 2.8 \\
\hline & & cut $1+0 \leq \cos \theta_{\ell j} \leq 1$ & 105.7 & 167.5 & 6.4 \\
\hline \multirow[t]{4}{*}{ BP3 } & \multirow[t]{2}{*}{2} & $\left|M_{l j}-M_{\phi}\right| \leq 10 \mathrm{GeV}$ & 154.1 & 1061.6 & 4.4 \\
\hline & & $\operatorname{cut} 1+(-0.9) \leq \cos \theta_{\ell j} \leq 1$ & 109.6 & 391.5 & 4.9 \\
\hline & \multirow[t]{2}{*}{3} & $\left|M_{l j}-M_{\phi}\right| \leq 10 \mathrm{GeV}$ & 62.5 & 815.0 & 2.1 \\
\hline & & $\operatorname{cut} 1+(-0.8) \leq \cos \theta_{\ell j} \leq 1$ & 60.1 & 254.7 & 3.4 \\
\hline
\end{tabular}




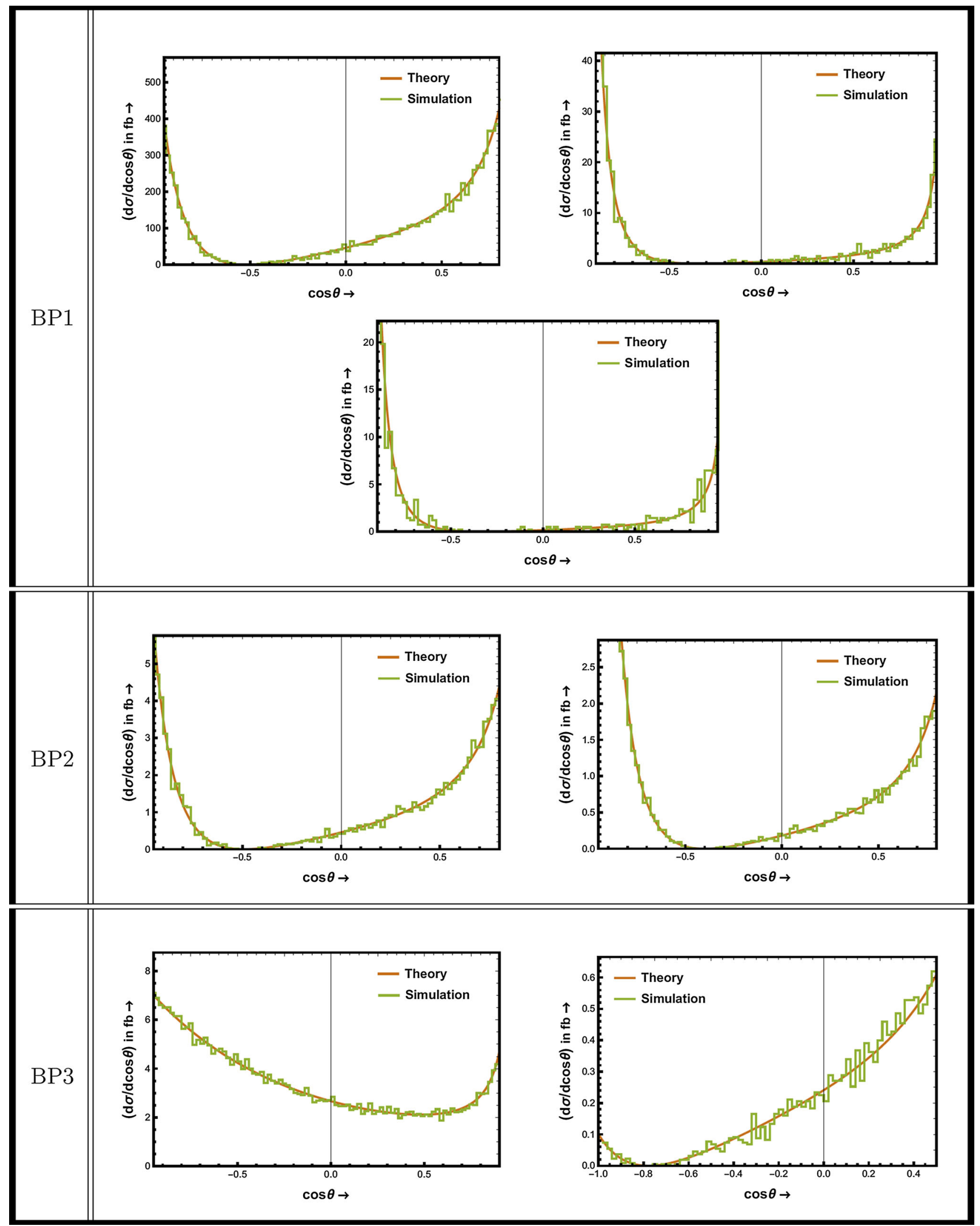

Fig. 12 Angular distribution for the production of $\left(\tilde{V}_{2 \mu}^{+1 / 3}\right)^{c}$ at various center of momentum energies for different benchmark points, arranged in the order of Table 12. The brown (smooth) curves indicate the theoretical expectations whereas the green (jagged) lines signify the PYTHIA simulated data 


\subsubsection{Leptoquark $\left(U_{3 \mu}^{+5 / 3}\right)^{c}$}

We present the PYTHIA analysis for leptoquark $\left(U_{3 \mu}^{+5 / 3}\right)^{c}$ in Table 13 with a luminosity of $100 \mathrm{fb}^{-1}$. By putting a cut on the invariant mass of lepton jet pair, we get the signals with very high significances $(602.2 \sigma, 648.7$ and $648.8 \sigma)$ in the case of BP1 for all the three center of momentum energies. The angular cut in this case becomes inessential. The leptoquark for BP2 can be reconstructed with the significances $18.8 \sigma$ and $21.1 \sigma$ at $\sqrt{s}$ to be $2 \mathrm{TeV}$ and $3 \mathrm{TeV}$, respectively. Using the angular cut, the significances can be upgraded to $26.5 \sigma$ and $29.5 \sigma$, respectively. For BP3 at $2 \mathrm{TeV}$, the cut of 10 $\mathrm{GeV}$ on $M_{\ell j}$ around the mass of leptoquark provides $4.6 \sigma$ significance for the signal events, whereas the angular cut of $(-0.9) \leq \cos \theta_{\ell j} \leq 1$ enhances it to $5.1 \sigma$. For the same benchmark point at $3 \mathrm{TeV}$, the significance for the signal events goes to $5.9 \sigma$ from $3.9 \sigma$ after applying the angular cut of $(-0.8) \leq \cos \theta_{\ell j} \leq 1$.

In Fig. 13, we show the angular distribution for the production of leptoquark $\left(U_{3 \mu}^{+5 / 3}\right)^{c}$ associated with a $u$-quark for all the three bench-mark points at different center of momentum energies. The brown (even) and green (uneven) lines signify the theoretical expectations and the PYTHIA simulated data, respectively. In this case, no zero in any of the distributions is found.

Table 13 Signal-background analysis for leptoquark $\left(U_{3 \mu}^{+5 / 3}\right)^{c}$ with luminosity $100 \mathrm{fb}^{-1}$ at $e-\gamma$ collider

\begin{tabular}{|c|c|c|c|c|c|}
\hline Bench-mark points & $\sqrt{s}$ in $\mathrm{TeV}$ & Cut & Signal & Back-ground & Signi-ficance \\
\hline \multirow[t]{6}{*}{ BP1 } & \multirow[t]{2}{*}{0.2} & $\left|M_{l j}-M_{\phi}\right| \leq 10 \mathrm{GeV}$ & 402140.1 & 43725.0 & 602.2 \\
\hline & & $\operatorname{cut} 1+(-0.2) \leq \cos \theta_{\ell j} \leq 1$ & 376284.9 & 32989.8 & 588.2 \\
\hline & \multirow[t]{2}{*}{2} & $\left|M_{l j}-M_{\phi}\right| \leq 10 \mathrm{GeV}$ & 421151.4 & 319.4 & 648.7 \\
\hline & & $\operatorname{cut} 1+(0.9) \leq \cos \theta_{\ell j} \leq 1$ & 268692.3 & 114.2 & 518.2 \\
\hline & \multirow[t]{2}{*}{3} & $\left|M_{l j}-M_{\phi}\right| \leq 10 \mathrm{GeV}$ & 421146.5 & 219.8 & 648.8 \\
\hline & & cut $1+(0.9) \leq \cos \theta_{\ell j} \leq 1$ & 247085.8 & 44.2 & 497.0 \\
\hline \multirow[t]{4}{*}{ BP2 } & \multirow[t]{2}{*}{2} & $\left|M_{l j}-M_{\phi}\right| \leq 10 \mathrm{GeV}$ & 1038.7 & 2003.6 & 18.8 \\
\hline & & $\operatorname{cut} 1+0 \leq \cos \theta_{\ell j} \leq 1$ & 814.4 & 129.1 & 26.5 \\
\hline & \multirow[t]{2}{*}{3} & $\left|M_{l j}-M_{\phi}\right| \leq 10 \mathrm{GeV}$ & 1110.4 & 1660.7 & 21.1 \\
\hline & & cut $1+0 \leq \cos \theta_{\ell j} \leq 1$ & 1014.0 & 167.5 & 29.5 \\
\hline \multirow[t]{4}{*}{ BP3 } & \multirow[t]{2}{*}{2} & $\left|M_{l j}-M_{\phi}\right| \leq 10 \mathrm{GeV}$ & 162.4 & 1061.6 & 4.6 \\
\hline & & $\operatorname{cut} 1+(-0.9) \leq \cos \theta_{\ell j} \leq 1$ & 115.5 & 391.5 & 5.1 \\
\hline & \multirow[t]{2}{*}{3} & $\left|M_{l j}-M_{\phi}\right| \leq 10 \mathrm{GeV}$ & 119.3 & 815.0 & 3.9 \\
\hline & & $\operatorname{cut} 1+(-0.8) \leq \cos \theta_{\ell j} \leq 1$ & 113.9 & 254.7 & 5.9 \\
\hline
\end{tabular}




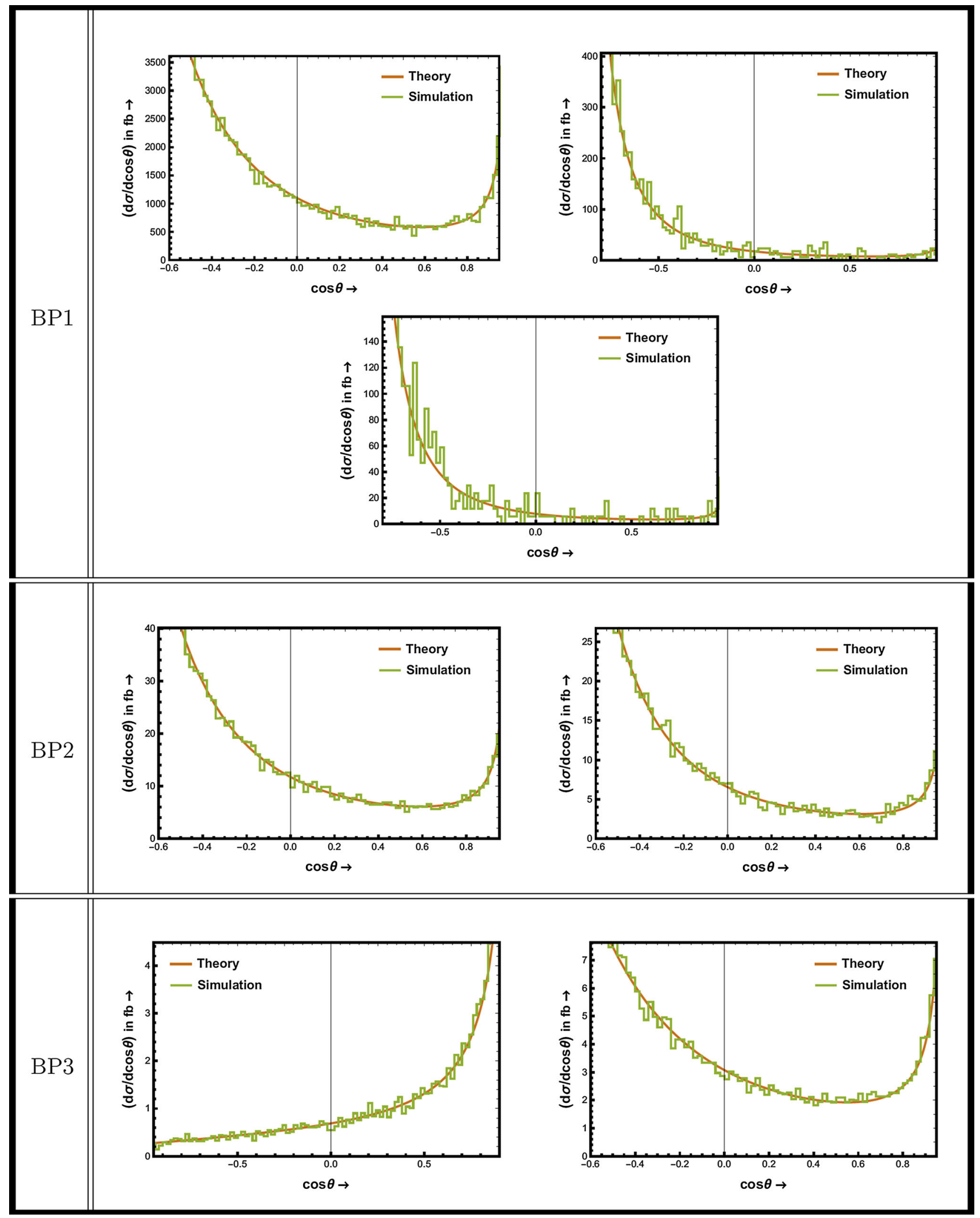

Fig. 13 Angular distribution for the production of $\left(U_{3 \mu}^{+5 / 3}\right)^{c}$ at various center of momentum energies for different benchmark points, arranged in the order of Table 13. The brown (smooth) curves indicate the theoretical expectations, whereas the green (jagged) lines signify the PYTHIA simulated data 


\section{Effects of non-monochromatic photons}

All of our simulations until this point were performed with monochromatic photon beams. However, the experimental collider technology developed so far cannot deal with monochromatic photons in the initial state. Rather the photons used in modern colliders have some energy distribution. The two ways for the production of these high energetic photons, which we are going to discuss, are laser backscattering and quasi-real photons emitted by fast charged leptons. For the latter process one can use protons instead of leptons, but it would make the environment messier due to the presence of a strongly interacting particle in the initial state, and therefore we stick to leptons only for the purposes of our discussion.

In the case of laser backscattering, a laser beam interacts with high energy electrons or positrons and thus highly photons produced in the backward direction due to Compton scattering. This facility will be available in linear $e^{+} e^{-}$colliders like CLIC [141] and ILC [142]. This option for the photon exists in ClacHEP, but not in MadGraph [143]. The distribution of photons in this case is given by [125]

$$
\begin{aligned}
\frac{1}{\sigma_{c}} & \frac{\mathrm{d} \sigma_{c}}{\mathrm{~d} y}=f(x, y) \\
= & \frac{2 \sigma_{0}}{x \sigma_{c}}\left[1-y+\frac{1}{1-y}-\frac{4 y}{x(1-y)}+\frac{4 y^{2}}{x^{2}(1-y)^{2}}\right] \\
& \quad \text { for } 0<y<y_{\max },
\end{aligned}
$$

where $y$ is the fraction of energy for the backscattered photon relative to the energy of the parent charged lepton, the maximum value of $y$ is $y_{\max }$, which can also be written as $\left(\frac{x}{1+x}\right)$, the constant $\sigma_{0}=\left(\pi e^{4} / m_{e}^{2}\right)$ with $e$ and $m_{e}$ being the electric charge and mass of the positron, respectively, and the total cross section for Compton scattering is given by

$\sigma_{c}=\frac{2 \sigma_{0}}{x}\left[\left(1-\frac{4}{x}-\frac{8}{x^{2}}\right) \ln (1+x)+\frac{1}{2}+\frac{8}{x}-\frac{1}{2(1+x)^{2}}\right]$.

If laser and positron with energies $\omega_{0}$ and $E$ collide at a small angle $\alpha_{0}$ for the backscattering, then the quantity $x$ is defined as $x=\left(4 E \omega_{0} / m_{e}^{2}\right) \cos ^{2}\left(\alpha_{0} / 2\right)$. However, the value of $x$ is taken as 4.82 in CalcHEP.

On the other hand, any fast moving charged particle can be considered as an electromagnetic radiation field by the equivalent photon approximation (EPA) [144-146]. This radiation can be interpreted as a flux of quasi-real photons with some energy distribution. Following the WilliamsWeizsäcker approximation, this distribution can be taken as

$$
\begin{aligned}
& f\left(y, q_{\max }^{2}\right) \\
& \quad=\frac{\alpha}{\pi}\left[\left(\frac{1}{y}-1+\frac{y}{2}\right) \ln \left(\frac{q_{\max }^{2}}{q_{\min }^{2}}\right)+\left(1-\frac{1}{y}\right)\left(1-\frac{q_{\min }^{2}}{q_{\max }^{2}}\right)\right]
\end{aligned}
$$

for $0<y<1$,

where $y$ is the fraction of the energy for the quasi-real photon with respect to that of the positron, $\alpha$ is the electromagnetic coupling constant, the minimum value for $q^{2}$ is $q_{\min }^{2}$, which can be expressed as $\left(\frac{m_{e}^{2} y^{2}}{1-y}\right)$, and the maximum value for $q^{2}$ is $q_{\max }^{2}$, which signifies the region for photon virtuality. It should be kept in mind that the four-momentum of a quasireal photon is denoted by $q^{\mu}$ in the lab frame. This scheme is available in both MadGraph and ClacHEP.

To visualize the effects of non-monochromatic photons on the zeros of the angular distribution for the production of the leptoquark associated with a quark (or antiquark) at an $e-\gamma$ collider, we pick four different scenarios, one from each of the leptoquark models having the zero inside the physical region. Two of them are taken from the lower energy and mass region and the other two are taken from the higher energy and mass region. These four scenarios are as follows: a) BP1 for $\left(S^{+1 / 3}\right)^{c}$ at $\sqrt{s}=0.2 \mathrm{TeV}$, b) BP2 for $\left(\tilde{R}_{2}^{+2 / 3}\right)^{c}$ at $\sqrt{s}=2 \mathrm{TeV}$ c) BP3 for $\left(U_{1 \mu}^{+2 / 3}\right)^{c}$ at $\sqrt{s}=2 \mathrm{TeV}$ and d) BP1 for $\left(\tilde{V}_{2 \mu}^{+1 / 3}\right)^{c}$ at $\sqrt{s}=0.2 \mathrm{TeV}$. The signal-background analysis and the cross section for production of leptoquarks in these four cases with laser backscattering and EPA are presented in Tables 14 and 15, respectively. It can easily be seen from these tables that the production cross section, the signal event and the significance get enhanced to a great extent by laser backscattering as compared to monochromatic photon beams, whereas the EPA scheme diminishes them notably. The increments in significances under laser backscattering for the above four scenarios are $83 \%, 47 \%, 11 \%$ and $40 \%$, respectively, whereas under EPA, the significances reduce by $27 \%, 65 \%, 91 \%$ and $51 \%$, respectively, for those cases. The production cross section and signal event for laser backscattering are slightly lower than for the monochromatic case in only the BP3 scenario for $\left(U_{1 \mu}^{+2 / 3}\right)^{c}$ at $\sqrt{s}=2 \mathrm{TeV}$. This occurs because of phase space suppression for low energy photons due to the heavy mass of leptoquark in the BP3 scenario.

The weighted differential distributions $\left(\frac{1}{\sigma} \cdot \frac{\mathrm{d} \sigma}{\mathrm{d} \cos \theta}\right)$ for the production of $\left(\tilde{V}_{2 \mu}^{+1 / 3}\right)^{c}$ in BP1 scenario at $\sqrt{s}=0.2 \mathrm{TeV}$ for laser backscattering, EPA and monochromatic photons, which are represented by orange, blue and green lines, respectively, are shown in the left panel of Fig. 14. As expected, the three distributions do not coincide. Still laser backscattering keeps us optimistic since it preserves the zero of the angular distribution (though slightly deviating from the monochromatic case). The slight shift of zero in this case is due to variation in $\sqrt{s}$ at each collision as caused by the distribution of the photon energy. But in the EPA scheme, the zero gets smeared off. The reason behind this smearing effect lies in the distribution for the transverse momentum of 
Table 14 Cross section for production of leptoquarks in the chosen four scenarios with laser backscattering, equivalent photon approximation and monochromatic photon at $e-\gamma$ collider

\begin{tabular}{|c|c|c|c|c|}
\hline \multirow[t]{2}{*}{ Photon } & \multicolumn{4}{|l|}{ Cross section in $\mathrm{fb}$} \\
\hline & $\left(S^{+1 / 3}\right)^{c}, \mathrm{BP} 1 \sqrt{s}=0.2 \mathrm{TeV}$ & $\left(\tilde{R}_{2}^{+2 / 3}\right)^{c}, \mathrm{BP} 2 \sqrt{s}=2 \mathrm{TeV}$ & $\left(U_{1 \mu}^{+2 / 3}\right)^{c}, \mathrm{BP} 3 \sqrt{s}=2 \mathrm{TeV}$ & $\left(\tilde{V}_{2 \mu}^{+1 / 3}\right)^{c}, \mathrm{BP} 1 \sqrt{s}=0.2 \mathrm{TeV}$ \\
\hline Laser backscattering & 688.20 & 4.87 & 11.11 & 3337.54 \\
\hline EPA & 101.42 & 0.81 & 0.40 & 486.94 \\
\hline Monochromatic & 430.24 & 2.89 & 14.84 & 2127.02 \\
\hline
\end{tabular}

Table 15 Signal-background analysis for chosen four scenarios with laser backscattering, equivalent photon approximation and monochromatic photon at $e-\gamma$ collider of luminosity $100 \mathrm{fb}^{-1}$. The term "cut1" indicates the invariant mass cut $\left|M_{l j}-M_{\phi}\right| \leq 10 \mathrm{GeV}$ whereas the term "cut2" denotes the angular cut corresponding to a particular benchmark point and center of momentum energy as shown in the other tables for signal-background analysis

\begin{tabular}{|c|c|c|c|c|c|c|c|}
\hline Model & Benchmark points & $\sqrt{s}$ in $\mathrm{TeV}$ & Photon & Cut & Signal & Background & Significance \\
\hline \multirow[t]{6}{*}{$\left(S_{1}^{+1 / 3}\right)^{c}$} & \multirow[t]{6}{*}{ BP1 } & \multirow[t]{6}{*}{0.2} & \multirow[t]{2}{*}{ Laser Backscattering } & Cut1 & 18518.7 & 36417.9 & 79.0 \\
\hline & & & & Cut $1+$ Cut 2 & 17499.5 & 18173.8 & 92.65 \\
\hline & & & \multirow[t]{2}{*}{ EPA } & Cut1 & 2863.6 & 1964.4 & 41.2 \\
\hline & & & & Cut $1+$ Cut 2 & 2051.6 & 1038.2 & 36.9 \\
\hline & & & \multirow[t]{2}{*}{ Monochromatic } & Cut1 & 11133.6 & 43725.0 & 47.5 \\
\hline & & & & Cut $1+$ Cut 2 & 10537.8 & 32989.8 & 50.5 \\
\hline \multirow[t]{6}{*}{$\left(\tilde{R}_{2}^{+2 / 3}\right)^{c}$} & \multirow[t]{6}{*}{$\mathrm{BP} 2$} & \multirow[t]{6}{*}{2} & \multirow[t]{2}{*}{ Laser Backscattering } & Cut1 & 62.7 & 2078.4 & 1.4 \\
\hline & & & & Cut $1+$ Cut 2 & 48.1 & 326.1 & 2.5 \\
\hline & & & \multirow[t]{2}{*}{ EPA } & Cut1 & 11.7 & 390.2 & 0.6 \\
\hline & & & & Cut $1+$ Cut 2 & 7.0 & 230.8 & 0.5 \\
\hline & & & \multirow[t]{2}{*}{ Monochromatic } & Cut1 & 27.0 & 2003.6 & 0.6 \\
\hline & & & & Cut $1+$ cut 2 & 20.8 & 129.1 & 1.7 \\
\hline \multirow[t]{6}{*}{$\left(U_{1 \mu}^{+2 / 3}\right)^{c}$} & \multirow[t]{6}{*}{ BP3 } & \multirow[t]{6}{*}{2} & \multirow[t]{2}{*}{ Laser Backscattering } & Cut1 & 131.8 & 446.3 & 5.5 \\
\hline & & & & Cut $1+$ Cut 2 & 93.4 & 246.0 & 5.1 \\
\hline & & & \multirow[t]{2}{*}{ EPA } & Cut1 & 4.5 & 86.1 & 0.5 \\
\hline & & & & Cut $1+$ Cut 2 & 3.5 & 79.7 & 0.4 \\
\hline & & & \multirow[t]{2}{*}{ Monochromatic } & Cut1 & 144.4 & 1061.6 & 4.2 \\
\hline & & & & Cut $1+$ Cut 2 & 102.2 & 391.5 & 4.6 \\
\hline \multirow[t]{6}{*}{$\left(\tilde{V}_{2 \mu}^{+1 / 3}\right)^{c}$} & \multirow[t]{6}{*}{ BP1 } & \multirow[t]{6}{*}{0.2} & \multirow[t]{2}{*}{ Laser Backscattering } & Cut1 & 168135.3 & 36417.9 & 371.8 \\
\hline & & & & Cut $1+$ Cut 2 & 157708.8 & 18173.8 & 376.0 \\
\hline & & & \multirow[t]{2}{*}{ EPA } & Cut1 & 24697.2 & 1964.4 & 151.3 \\
\hline & & & & Cut $1+$ Cut 2 & 17881.0 & 1038.2 & 130.0 \\
\hline & & & \multirow[t]{2}{*}{ Monochromatic } & Cut1 & 102107.7 & 43725.0 & 267.4 \\
\hline & & & & Cut $1+$ Cut 2 & 96573.2 & 32989.8 & 268.3 \\
\hline
\end{tabular}

photons $\left(p_{T}^{\gamma}\right)$ which is depicted in the right panel of Fig. 14 . Although most of the photons in EPA have small $p_{T}^{\gamma}$, there is a non-zero possibility for them to acquire very high $p_{T}^{\gamma}$ too. As can be seen from the right panel of Fig. 14, the highest $p_{T}^{\gamma}$ achieved by photons from the $100 \mathrm{GeV}$ positron under EPA is around $90 \mathrm{GeV}$. Due to this non-zero $p_{T}^{\gamma}$ the direction of the photons changes in the case of each collision and hence $e$ and $\gamma$ no longer move collinearly in opposite directions.
Another important point to mention is that here we have presented the angular distribution for all the cases in terms of angle between electron and leptoquark; however, one can use the distribution in terms of the angle between photon and leptoquark too. In the case of monochromatic photons, the system lies in the center of momentum frame and the two angles mentioned above are supplementary to each other; hence the distributions with respect to them are equivalent, 


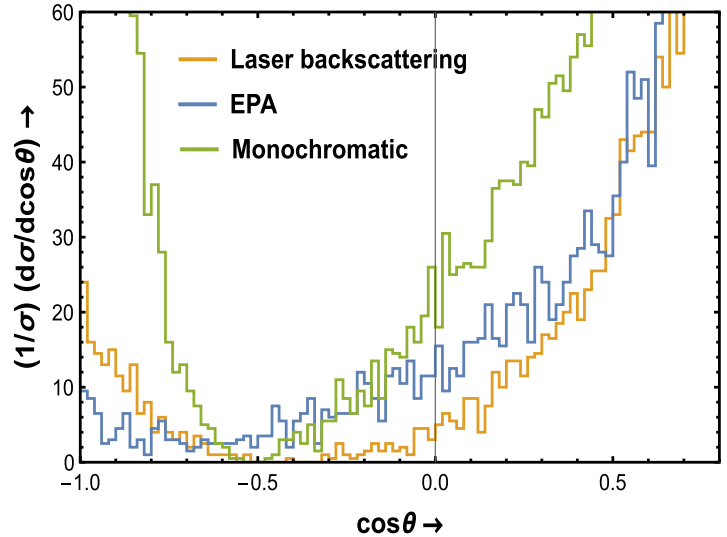

Fig. 14 The comparison among laser backscattering, EPA and monochromatic photons (represented by orange, blue and green lines, respectively) in terms of weighted differential distribution $\left(\frac{1}{\sigma} \cdot \frac{\mathrm{d} \sigma}{\mathrm{d} \cos \theta}\right)$

apart from a negative sign. For laser backscattering, the system no longer lies in the center of momentum frame due to the varying energy of the photons; however, the abovementioned two angles still remain supplementary to each other since all the photons are found with zero $p_{T}^{\gamma}$ only and therefore, the two distributions look quite similar discarding the negative sign. But in the EPA scenario, neither the system lies in the center of momentum frame nor the angle between photon-leptoquark and electron-leptoquark remains supplementary; hence the angular distributions with respect to these two angles disagree conspicuously. Talking in terms of zeros of angular distributions, the situations are worse while considering the angle between photon and leptoquark under EPA. A similar kind of things happens for other leptoquark models with different benchmark point and center of momentum energy.

\section{Conclusion}

In conclusion, we have studied the zeros of the single-photon tree-level amplitude at the $e-\gamma$ collider producing a leptoquark associated with a quark (or antiquark). Unlike other colliders, we find that the position of zeros of the singlephoton tree-level amplitude in this case does depend on the center of momentum energy and on the mass and charge of the leptoquark. The cosine of the angle between leptoquark and initial state electron, at which zero of the angular distribution happens, approaches $\pm 1 / 3$ asymptotically depending on the charge of the produced leptoquark for very high value of $\sqrt{s}$ with respect to the mass of leptoquark. No zero in the differential distribution can be found for leptoquarks having charges smaller than -1 unit. In a PYTHIA based analysis we look for both light and heavy leptoquarks

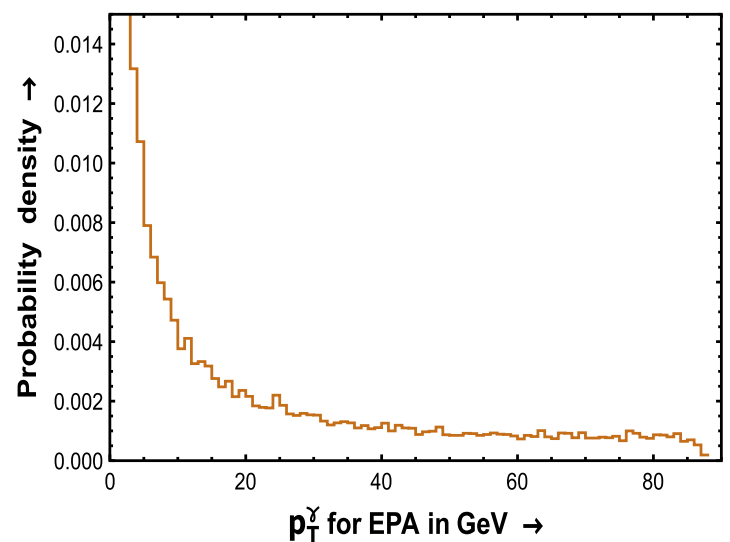

for the production of $\left(\tilde{V}_{2 \mu}^{+1 / 3}\right)^{c}$ in the BP1 scenario at $\sqrt{s}=0.2 \mathrm{TeV}$ is shown in the left panel. The distribution for the transverse momentum of photon from a $100 \mathrm{GeV}$ positron under the EPA scheme is shown in the right panel

at both low and high energy scales. The scenario of light leptoquarks having small couplings to quarks and leptons of all generations is not completely ruled out by Tevatron. In our simulation, we reconstruct the leptoquark from the lepton-jet pair and then study the differential distribution against the cosine of the angle between it and the initial state electron which matches with the theoretical expectation. We have also studied the consequences of using nonmonochromatic photons for the production of leptoquarks at electron-photon colliders. The production cross section and significance increase notably under laser backscattering and decrease terribly under the equivalent photon approximation. It turns out that a non-zero transverse momentum of photons smears off the zeros of the angular distributions completely in the equivalent photon approximation whereas laser backscattering preserves them (though slightly deviating from the monochromatic case) since all the photons here move only in the direction opposite to the electron. It seems that laser backscattering is very promising for investigating the production of leptoquarks at an $e-\gamma$ collider by means of the zeros of the differential distribution.

Acknowledgements The authors thank Abhay Deshpande, Yoshitaka Kuno, Saurabh Sandilya and Vishal Bhardwaj for some useful discussions. The authors also thank Alexander Pukhov, Olivier Mattelaer and Antonio Costantini for private communications. PB and AK also thank SERB India, grant no: CRG/2018/004971 for the financial support.

Data Availability Statement This manuscript has no associated data or the data will not be deposited. [Authors' comment: Electron-photon collider is a proposed experiment. The results of this paper are basically theoretical predictions simulated by PYTHIA and hence they need to be confirmed in the future experiments. We have not analysed any astrophysical or experimental data in this paper.] 
Open Access This article is licensed under a Creative Commons Attribution 4.0 International License, which permits use, sharing, adaptation, distribution and reproduction in any medium or format, as long as you give appropriate credit to the original author(s) and the source, provide a link to the Creative Commons licence, and indicate if changes were made. The images or other third party material in this article are included in the article's Creative Commons licence, unless indicated otherwise in a credit line to the material. If material is not included in the article's Creative Commons licence and your intended use is not permitted by statutory regulation or exceeds the permitted use, you will need to obtain permission directly from the copyright holder. To view a copy of this licence, visit http://creativecomm ons.org/licenses/by/4.0/.

Funded by SCOAP ${ }^{3}$.

\section{References}

1. C. Jogesh, Pati and Abdus Salam, Lepton number as the fourth color. Phys. Rev. D 10, 275 (1974)

2. H. Georgi, S.L. Glashow, Unity of all elementary - particle forces. Phys. Rev. Lett. 32, 438 (1974)

3. H. Georgi, The state of art - gauge theories. AIP Conf. Proc. 23, 575 (1975)

4. H. Fritzsch, P. Minkowski, Unified interactions of leptons and hadrons. Ann. Phys. 93, 193 (1975)

5. E. Farhi, L. Susskind, Technicolour. Phys. Rep. 74, 277 (1981)

6. K. Lane, M. Ramana, Walking technicolor signatures at hadron colliders. Phys. Rev. D 44, 2678 (1991)

7. W. Buchmüller, R. Rückl, D. Wyler, Leptoquarks in LeptonQuark Collisions. Phys. Lett. B 191, 442-448 (1987)

8. J. Blümlein, R. Rückl, Production of scalar and vector leptoquarks in $e^{+} e$ annihilation. Phys. Lett. B 304, 337-346 (1993)

9. I. Doršner, S. Fajfer, A. Greljo, J.F. Kamenik, N. Košnik, Physics of leptoquarks in precision experiments and at particle colliders. Phys. Rep. 641 (2016)

10. A. Belyaev, C. Leroy, R. Mehdiyev, A. Pukhov, Leptoquark single and pair production at LHC with CalcHEP/CompHEP in the complete model. JHEP 0509, 005 (2005)

11. M. Leurer, A comprehensive study of Leptoquark bounds. Phys. Rev. D 49, 333-342 (1994)

12. S. Davidson, D. Bailey, B.A. Campbell, Model independent constraints on leptoquarks from rare processes. Z. Phys. C 61, 613644 (1994)

13. J.P. Lees et al. [BaBar Collaboration], Evidence for an excess of $\bar{B} \rightarrow D^{(*)} \tau^{-} \bar{\nu}_{\tau}$. Phys. Rev. Lett. 109, 101802 (2012)

14. A. Matyja et al. [Belle Collaboration], Observation of $B^{0} \rightarrow$ $D^{*-} \tau^{+} v_{\tau}$ decay at Belle. Phys. Rev. Lett. 99, 191807 (2007)

15. R. Aaij et al. [LHCb Collaboration], Measurement of the ratio of branching fractions $B\left(B^{0} \rightarrow D^{*+} \tau^{-} \bar{v}_{\tau}\right) / B\left(B^{0} \rightarrow D^{*+} \mu^{-} \bar{v}_{\mu}\right)$. Phys. Rev. Lett. 115, 111803 (2015)

16. R. Aaij et al. [LHCb Collaboration], Test of lepton universality using $B^{+} \rightarrow K^{+} l^{+} l^{-}$. Phys. Rev. Lett. 113, 151601 (2014)

17. R. Aaij et al. [LHCb Collaboration], Angular analysis of the $B^{0} \rightarrow K^{* 0} \mu^{+} \mu^{-}$decay using $3 \mathrm{fb}^{-1}$ of integrated luminosity. JHEP 1602, 104 (2016)

18. G.W. Bennett et al. [Muon g-2 Collaboration], Measurement of the negative muon anomalous magnetic moment to $0.7 \mathrm{ppm}$. Phys. Rev. Lett. 92, 161802 (2004)

19. G.W. Bennett et al. [Muon g-2 Collaboration], Final report of the E821 muon anomalous magnetic moment measurement at BNL. Phys. Rev. D 73, 072003 (2006)

20. V. Khachatryan et al. [CMS Collaboration], Search for leptonflavour-violating decays of the Higgs boson. Phys. Lett. B 749, 337 (2015)
21. N. Košnik, D. Bečirević, I. Doršner, S. Fajfer, D.A. Faroughy, O. Sumensari, Ultraviolet complete Leptoquark scenario addressing the B physics anomalies. Springer Proc. Phys. 234, 425-430 (2019)

22. I. Doršner, S Fajfer, O. Sumensari, Muon g-2 and scalar leptoquark mixing. arXiv:1910.03877 [hep-ph]

23. D. Bečirević, I. Doršner, S. Fajfer, N. Košnik, D.A. Faroughy, O. Sumensari, Scalar leptoquarks from grand unified theories to accommodate the B-physics anomalies. Phys. Rev. D 98(5), 055003 (2018)

24. D. Bečirević, S. Fajfer, N. Košnik, O. Sumensari, Leptoquark model to explain the $B$-physics anomalies, $R_{K}$ and $R_{D}$. Phys. Rev. D 94(11), 115021 (2016)

25. S. Fajfer, N. Košnik, Vector leptoquark resolution of $R_{K}$ and $R_{D^{(*)}}$ puzzles. Phys. Lett. B 755, 270-274 (2016)

26. I. Doršner, S. Fajfer, N. Košnik, Leptoquark mechanism of neutrino masses within the grand unification framework. Eur. Phys. J. C 77(6), 417 (2017)

27. S. Fajfer, N. Košnik, L. Vale Silva, Footprints of leptoquarks: from $R_{K^{(*)}}$ to $K \rightarrow \pi \nu \bar{v}$. Eur. Phys. J. C 78(4), 275 (2018)

28. P. Bandyopadhyay, R. Mandal, Vacuum stability in an extended standard model with a leptoquark. Phys. Rev. D 95(3), 035007 (2017)

29. U. Aydemir, T. Mandal, S. Mitra, Addressing the $R_{D^{(*)}}$ anomalies with an $S_{1}$ leptoquark from $\mathrm{SO}(10)$ grand unification. Phys. Rev. D 101(1), 015011 (2020)

30. S. Sahoo, R. Mohanta, A.K. Giri, Explaining the $R_{K}$ and $R_{D^{*}}$ anomalies with vector leptoquarks. Phys. Rev. D 95(3), 035027 (2017)

31. S. Sahoo, R. Mohanta, Impact of vector leptoquark on $\bar{B} \rightarrow$ $\bar{K}^{*} l^{+} l$ anomalies. J. Phys. G 45(8), 085003 (2018)

32. S. Sahoo, R. Mohanta, Study of the rare semileptonic decays $B_{d}^{0} \rightarrow K^{*} l^{+} l$ in scalar leptoquark model. Phys. Rev. D 93(3), 034018 (2016)

33. M. Duraisamy, S. Sahoo, R. Mohanta, Rare semileptonic $B \rightarrow$ $K(\pi) l_{i}^{-} l_{j}^{+}$decay in vector leptoquark model. Phys. Rev. D 95(3), 035022 (2017)

34. S. Sahoo, R. Mohanta, Effects of scalar leptoquark on semileptonic $\Lambda_{b}$ decays. New J. Phys. 18(9), 093051 (2016)

35. R. Mohanta, Effects of scalar leptoquarks on the decays of $B_{s}$ meson. Phys. Rev. D 89(1), 014020 (2014)

36. M. Bauer, M. Neubert, Minimal leptoquark explanation for the $R_{D^{(*)}}, R_{K}$, and $(g-2)_{\mu}$ anomalies. Phys. Rev. Lett. 116(14), 141802 (2016)

37. R. Barbieri, C.W. Murphy, F. Senia, B-decay anomalies in a composite leptoquark model. Eur. Phys. J. C 77(1), 8 (2017)

38. N.G. Deshpande, B. Dutta, Leptoquark explanation of HERA anomaly in the context of gauge unification. Phys. Lett. B 424, 313-321 (1998)

39. D. Buttazzo, A. Greljo, G. Isidoria, D. Marzoccaa, B-physics anomalies: a guide to combined explanations. JHEP 11, 044 (2017)

40. K. Cheung, Muon anomalous magnetic moment and leptoquark solutions. Phys. Rev. D 64, 033001 (2001)

41. D. Das, K. Ghosh, M. Mitra, S. Mandal, Probing sterile neutrinos in the framework of inverse seesaw mechanism through leptoquark productions. Phys. Rev. D 97, 015024 (2018)

42. T. Mandal, S. Mitra, S. Raz, $R_{D^{(*)}}$ motivated $\mathscr{S}_{1}$ leptoquark scenarios: impact of interference on the exclusion limits from LHC data. Phys. Rev. D 99(5), 055028 (2019)

43. N. Assad, B. Fornal, B. Grinstein, Baryon number and Lepton universality violation in leptoquark and diquark models. Phys. Lett. B 777, 324-331 (2018)

44. B. Fornal, S.A. Gadam, B. Grinstein, Left-right SU(4) vector leptoquark model for flavor anomalies. Phys. Rev. D 99, 055025 (2019) 
45. E.C. Leskow, G. D'Ambrosio, A. Crivellin, D. Müller, $(g-2)_{\mu}$, lepton flavor violation, and $\mathrm{Z}$ decays with leptoquarks: correlations and future prospects. Phys. Rev. D 95, 055018 (2017)

46. A. Crivellin, D. Müller, T. Ota, Simultaneous explanation of $R\left(D^{(*)}\right)$ and $b \rightarrow s \mu^{+} \mu^{-}$: the last scalar leptoquarks standing. JHEP 1709, 040 (2017)

47. A. Crivellin, D. Müller, F. Saturnino, Flavour phenomenology of the leptoquark singlet-triplet model. arXiv:1912.04224 [hep-ph]

48. L. Calibbi, A. Crivellin, T. Li, Model of vector leptoquarks in view of B-physics anomalies. Phys. Rev. D 98(11), 115002 (2018)

49. A. Crivellin, C. Greub, D. Müller, F. Saturnino, Importance of loop effects in explaining the accumulated evidence for new physics in B decays with a vector leptoquark. Phys. Rev. Lett. 122(1), 011805 (2019)

50. J. Aebischer, A. Crivellin, C. Greub, QCD improved matching for semileptonic B decays with leptoquarks. Phys. Rev. D 99(5), 055002 (2019)

51. R. Mandal, Fermionic dark matter in leptoquark portal. Eur. Phys. J. C 78, 726 (2018)

52. W.-S. Hou, T. Modak, G.-G. Wong, Scalar leptoquark effects on $B \rightarrow \mu \bar{v}$ decay. Eur. Phys. J. C 79, 964 (2019)

53. C. Cornella, J. Fuentes-Martin, G. Isidori, Revisiting the vector leptoquark explanation of the B-physics anomalies. JHEP 07, 168 (2019)

54. P. Bandyopadhyay, R. Mandal, Revisiting scalar leptoquark at the LHC. Eur. Phys. J. C 78, 491 (2018)

55. A. Bhaskar, D. Das, B. De, S. Mitra, Enhancement of Higgs Production Through Leptoquarks at the LHC. arXiv:2002.12571 [hep-ph]

56. K. Chandak, T. Mandal, S. Mitra, Hunting for scalar leptoquarks with boosted tops and light leptons. Phys. Rev. D 100(7), 075019 (2019)

57. T. Mandal, S. Mitra, S. Seth, Single productions of colored particles at the LHC: an example with scalar leptoquarks. JHEP 1507, 028 (2015)

58. A. Alves, O.J.P. Éboli, G.G. di Cortona, R.R. Moreira, Indirect and monojet constraints on scalar leptoquarks. Phys. Rev. D 99, 095005 (2019)

59. I. Doršner, S. Fajfer, M. Patra, A comparative study of the $S_{1}$ and $U_{1}$ leptoquark effects in the light quark regime. Eur. Phys. J. C 80(3), 204 (2020)

60. R. Mandal, A. Pich, Constraints on scalar leptoquarks from lepton and kaon physics. JHEP 1912, 089 (2019)

61. S. Mandal, M. Mitra, N. Sinha, Probing leptoquark and heavy neutrino at LHeC. Phys. Rev. D 98, 095004 (2018)

62. R. Padhan, S. Mandal, M. Mitra, N. Sinha, Signatures of $\tilde{R}_{2}$ class of leptoquarks at upcoming ep colliders. arXiv:1912.07236 [hep$\mathrm{ph}]$

63. S. Davidson, S. Descotes-Genon, Minimal flavour violation for leptoquarks. JHEP 11, 073 (2010)

64. J. Fuentes-Martin, G. Isidori, M. König, N. Selimović, Vector leptoquarks beyond tree level. Phys. Rev. D 101(3), 035024 (2020)

65. M.J. Baker, J. Fuentes-Martin, G. Isidori, M. König, High$p_{T}$ signatures in vector-leptoquark models. Eur. Phys. J. C 79(4), 334 (2019)

66. G. Bhattacharyya, J.R. Ellis, K. Sridhar, Bounds on the masses and couplings of leptoquarks from leptonic partial widths of the Z. Phys. Lett. B 336, 100-106 (1994)

67. J.L. Hewett, T.G. Rizzo, Leptoquark-boson signals at $e^{+} e^{-}$colliders. Phys. Rev. D 36, 3367 (1987)

68. T. Plehn, H. Spiesberger, M. Spira, P.M. Zerwas, Formation and decay of scalar leptoquarks/squarks in ep collisions. Z. Phys. C 74, 611-614 (1997)

69. M. Krämer, T. Plehn, M. Spira, P.M. Zerwas, Pair production of scalar leptoquarks at the Fermilab Tevatron. Phys. Rev. Lett. 79, 341-344 (1997)
70. AMY Collaboration (G.N. Kim et al.), A search for leptoquark and colored lepton pair production in $e^{+} e^{-}$annihilations at TRISTAN. Phys. Lett. B 240, 243-249 (1990)

71. G. Alexander et al. [OPAL collaboration], A search for scalar leptoquarks in Z0 deacys. Phys. Lett. B 263, 123-134 (1991)

72. B. Adeva et al. [L3 collaboration], search for scalar leptoquarks in Z0 deacys. Phys. Lett. B 261, 169-176 (1991)

73. UA2 Collaboration, A search for scalar leptoquarks at the CERN $\bar{p} p$ collider. Phys. Lett. B 274, 507-512 (1992)

74. DELPHI Collaboration, Search for single leptoquark production in $e^{+} e$ collisions up to $\sqrt{s}=208 \mathrm{GeV}$ with the DELPHI detector, DELPHI 2001-080 CONF 508

75. Kaori Maeshima for the CDF Collaboration, Leptoquark Searches at the Tevatron, FERMILAB-Conf-96, 413-E

76. The ATLAS Collaboration, Search for scalar leptoquarks in $p p$ collisions at $\sqrt{s}=13 \mathrm{TeV}$ with the ATLAS experiment. N. J. Phys. 18, 093016 (2016)

77. The ATLAS Collaboration, Searches for third-generation scalar leptoquarks in $\sqrt{s}=13 \mathrm{TeV} p p$ collisions with the ATLAS detector. JHEP 06, 144 (2019)

78. The CMS Collaboration, Search for third-generation scalar leptoquarks decaying to a top quark and $\tau$ lepton at $\sqrt{s}=13 \mathrm{TeV}$. Eur. Phys. J. C 78, 707 (2018)

79. D.E. Acosta, S.K. Blessing, Leptoquark searches at HERA and the Tevatron. Ann. Rev. Nucl. Part. Sci. 49, 389-434 (1999)

80. ZEUS Collaboration (S. Chekanov et al.), A search for resonance decays to lepton + jet at HERA and limits on leptoquarks. Phys. Rev. D 68, 052004 (2003)

81. H1 Collaboration, Search for Leptoquark Bosons in ep Collisions at HERA. Phys. Lett. B 629, 9-19 (2005)

82. G. Wang, Leptoquark searches at the Tevatron. AIP Conf. Proc. 407, 345 (1997)

83. Thomas Nunnemann (on behalf of the D0 Collaboration), Searches for Leptoquark Production at D0. arXiv:0710.0255 [hepex]

84. K.O. Mikaelian, M.A. Samuel, D. Sahdev, Magnetic moment of weak bosons produced in $p p$ and $p \bar{p}$ collisions. Phys. Rev. Lett. 43, 746 (1979)

85. C.J. Goebel, F. Halzen, J.P. Leveille, Angular zeros of Brown, Mikaelian, Sahdev, and Samuel and the factorization of tree amplitudes in gauge theories. Phys. Rev. D 23, 2682 (1981)

86. Z. Dongpei, Zeros in scattering amplitudes and the structure of non-Abelian gauge theories. Phys. Rev. D 22, 2266 (1980)

87. S.J. Brodsky, R.W. Brown, Zeros in amplitudes: gauge theory and radiation interference. Phys. Rev. Lett. 49, 966 (1982)

88. K.O. Mikelian, Zeros in energy and angular distributions with real or virtual photons. Phys. Rev. D 25, 66 (1982)

89. K.O. Mikelian, Angular distribution of $\mathrm{W}$ bosons in hadron hadron $\rightarrow W^{ \pm} \gamma X$. Phys. Rev. D 26, 1085-1089 (1982)

90. M.L. Laursen, M.A. Samuel, A. Sen, Amplitude zeros in $p \bar{p}$ collisions and the quark magnetic moment. Phys. Rev. D 26, 2535 (1982)

91. M.L. Laursen, M.A. Samuel, A. Sen, G. Tupper, Do amplitude zeros persists in higher order? Nucl. Phys. B 226, 429-436 (1983)

92. R.W. Brown, K.L. Kowalski, S.J. Brodsky, Classical radiation zeros in gauge theory amplitudes. Phys. Rev. D 28, 624 (1983)

93. R.W. Robinett, The $\mathrm{W}$ magnetic moment in electroweak mixing and the composite models and amplitude zeros in $q_{i} \bar{q}_{j} \rightarrow W \gamma$. Phys. Rev. D 28, 1185 (1983)

94. G. Passarino, Physical null zones and radiation representation. Nucl. Phys. B 224, 265-288 (1983)

95. M.A. Samuel, Amplitude zeros. Phys. Rev. D 27, 2724-2731 (1983)

96. M.L. Laursen, M.A. Samuel, A. Sen, On the spoiling of amplitude (radiation) zeros at the one loop level and infrared finiteness. Phys. Rev. D 28, 650 (1983) 
97. M.L. Laursen, M.A. Samuel, A. Sen, Radiation zeros and a test for the $g$ values of the $\tau$ lepton. Phys. Rev. D 29, 2652-2654 (1984)

98. K. Hagiwara, F. Halzen, F. Herzog, Jets in $p \bar{p}$ collisions: radiation zeros and the electric charges of coloured quarks. Phys. Lett. B 135, 324 (1984)

99. M.A. Samuel, A. Sen, G.S. Sylvester, M.L. Laursen, General criteria for radiation amplitude zeros. Phys. Rev. D 29, 994 (1984)

100. G. Passarino, Radiation zeros and gravity. Nucl. Phys. B 241 , 48-60 (1984)

101. R.W. Robinett, A class of supersymmetric radiation zeros. Phys. Rev. D 30, 688 (1984)

102. R.W. Brown, K. Kowalski, Szeros. Phys. Lett. B 144, 235-239 (1984)

103. R.W. Brown, Understanding something about nothing: radiation zeros. AIP Conf. Proc. 350, 261-272 (1995)

104. J.D. Stroughair, C.L. Bilachak, The determiantion of the W anomalous magnetic moment in $p \bar{p} \rightarrow W \gamma X$. Z. Phys. C 29, 415-419 (1984)

105. J.H. Reid, A. Sen, On the factorization of collinear and infrared singularities in qcd corrections to the Mikaelian zero. Prog. Theor. Phys. 75, 98 (1986)

106. J.H. Reid, G. Tupper, M. van Zijl, A study of the amplitude zero in $W^{-} \rightarrow$ jet + jet $+\gamma$ using the Lund Model. Phys. Lett. B 218, 473 (1989)

107. M.A. Samuel, M. Frank, C. Hamzaoui, D. Pouliot, K.B. Samuel, $\mathrm{G}$. Li, On the uniqueness of the radiation amplitude zero in radiative W decay. Phys. Lett. B 243, 293-295 (1990)

108. G. Couture, Radiative zeros and extra neutral gauge bosons at Large Hadron Colliders. Phys. Rev. D 39, 2527-2535 (1989)

109. M.A. Samuel, G. Li, N. Sinha, R. Sinha, M.K. Sundersan, The magnetic moment of the $\mathrm{W}$ boson. Phys. Lett. B 280, 124-128 (1992)

110. M.A. Samuel, N. Sinha, R. Sinha, M.K. Sundersan, W radiative decays and the determination of magnetic dipole and electric quadrupole moments of the W. Phys. Rev. D 44, 2064 (1991)

111. M.A. Samuel, G. Li, N. Sinha, R. Sinha, M.K. Sundersan, Bounds on the magnetic moment of the W boson. Phys. Rev. Lett. 67, 9-11 (1991)

112. X.G. He, H. Lew, Scattering amplitude zero in $d \bar{u} \rightarrow \gamma H$ sup. Mod. Phys. Lett. A 3, 1199-1203 (1988)

113. M.A. Doncheski, F. Halzen, Observable radiation zer in HERA intreactions. Z. Phys. C 52, 673-676 (1991)

114. M. Heyssler, W.J. Stirling, Radiation zeros at HERA, more about nothing. Eur. Phys. J. C 4, 289-299 (1998)

115. F. Mamedov, Weak Boson production amplitude zeros, equalities of the helicity amplitudes. Phys. Rev. D 66, 033004 (2002)

116. D0 Collaboration, First study of the radiation-amplitude zero in $W \gamma$ production and limits on anomalous $W W \gamma$ couplings at $\sqrt{s}=1.96$ TeV. Phys. Rev. Lett. 100, 241805 (2008)

117. K. Hagiwara, T. Yamada, Null radiation zone at LHC. Phys. Rev. D 87(1), 014021 (2013)

118. B. Badelek et al., The photon collider at TESLA. Int. J. Mod. Phys. A 19, 5097-5186 (2004)

119. V. Telnov, Photon collider at TESLA: parameters and interaction region issues. arXiv:hep-ex/0101002

120. V. Telnov, Photon collider Higgs factories. J. Instrum. 9(09), C09020 (2014)

121. V. Telnov, Photon collider at TESLA. Nucl. Instrum. Methods A 472, 43-60 (2001)

122. V. Telnov, Status of gamma-gamma, gamma-electron colliders. Nucl. Phys. B (Proc. Suppl.) 82, 359-366 (2000)

123. V. Telnov, Prospects of high energy photon colliders. Nucl. Part. Phys. Proc. 273-275, 219-224 (2016
)

124. I.F. Ginzburg et al., Production of high-energy colliding $\gamma \gamma$ and $\gamma e$ beams with a high luminosity at VLEPP accelerators. Pis'ma Zh. Eksp. Teor. Fiz. 34(9), 514-518 (1981)

125. I.F. Ginzburg et al., Colliding $\gamma e$ and $\gamma \gamma$ beams based on the single-pass $e^{+} e^{-}$colliders (VLEPP type). Nucl. Instrum. Methods Phys. Res. 205(1-2), 47-68 (1981)

126. I.F. Ginzburg et al., An Interaction Region for GammaGamma and Gamma-Electron Collisions at TESLA/SBLC. arXiv:hep-ex/9707017v1

127. M.M. Velasco et al., Photon-photon and electron-photon colliders with energies below a TeV, SLAC ECONF C010630 E3005

128. F. Cuypers, Leptoquark production in electron photon scattering. Nucl. Phys. B 474, 57-71 (1996)

129. J.E.C. Montalvo, J.P. Éboli, Composite vector leptoquarks in $e^{+} e^{-}, \gamma e$ and $\gamma \gamma$ colliders. Phys. Rev. D 47, 837 (1993)

130. O.J.P. Eboli, E.M. Gregores, M.B. Margo, P.G. Mercadante, S.F. Novaes, Searching for leptoquarks in electron photon colliders. Phys. Lett. B 311, 147-152 (1993)

131. N.G. Deshpande, X.-G. He, S. Oh, Amplitude zeros in radiative decays of scalar particles. Phys. Rev. D 51, 2295-2301 (1995)

132. M.A. Doncheski, Robinett, Radiation zeros and scalar particles beyond the standard model. Phys. Lett. B 435, 364-372 (1998)

133. H. Nadeau, D. London, Leptoquarks at $e \gamma$ colliders. Phys. Rev. D 47, 3742 (1993)

134. M. Leurer, Acomprehensive study of leptoquark bounds. Phys. Rev. D 49, 333-342 (1994)

135. M. Leurer, Bounds on vector leptoquarks. Phys. Rev. D 50, 536541 (1994)

136. M. Tanabashi et al. [Particle Data Group], Phys. Rev. D 98, 030001 (2018)

137. A. Belyaev, N.D. Christensen, A. Pukhov, Comput. Phys. Commun. 184, 1729 (2013). https://doi.org/10.1016/j.cpc.2013.01. 014. arXiv:1207.6082 [hep-ph]

138. F. Staub, Comput. Phys. Commun. 185, 1773 (2014). arXiv:1309.7223 [hep-ph]

139. T. Sjostrand, L. Lonnblad, S. Mrenna. arXiv:hep-ph/0108264

140. M. Cacciari, G.P. Salam, G. Soyez, Eur. Phys. J. C 72, 1896 (2012). https://doi.org/10.1140/epjc/s10052-012-1896-2. [arXiv:1111.6097 [hep-ph]]

141. CLIC collaboration. clic-study.web.cern.ch

142. ILC collaboration. ilchome.web.cern.ch

143. J. Alwall, R. Frederix, S. Frixione, V. Hirschi, F. Maltoni, O. Mattelaer, H.S. Shao, T. Stelzer, P. Torrielli, M. Zaro, The automated computation of tree-level and next-to-leading order differential cross sections, and their matching to parton shower simulations. JHEP 07, 079 (2014)

144. V.M. Budnev, I.F. Ginzburg, G.V. Meledin, V.G. Serbo, The twophoton particle production mechanism; physical problems. Applications. Equivalent photon approximation. Phys. Rep. 15, 181$281(1975)$

145. E.J. Williams, Kgl. Danske Vidensk. Selskab. Mat.-Fiz. Medd 13, N4 (1935)

146. K.F. von Weizsäcker, Z. Phys. 88, 612 (1934) 